July 2016

\title{
"Using the Salop Circle to Study Scale Effects in Schumpeterian Growth Models: Why Inter-sectoral Knowledge Diffusion Matters"
}

Elie Gray, André Grimaud 


\title{
Using the Salop Circle to Study Scale Effects in Schumpeterian Growth Models: Why Inter-sectoral Knowledge Diffusion Matters
}

\author{
Elie Gray, André Grimaud ${ }^{\dagger}$
}

Working paper - This version, June 2016

\begin{abstract}
This paper analyzes the link between the fact that fully endogenous growth models exhibit (or not) the non-desirable scale effects property and assumptions regarding the intensity of knowledge diffusion. In that respect, we extend a standard Schumpeterian growth model by introducing explicitly knowledge diffusion over a Salop (1979) circle: a continuum of sectors simultaneously sending and receiving knowledge is located over the circle.

The link between knowledge diffusion and scale effects stems from the fact that the more diffusion spreads with the size of the economy, the larger the pools of knowledge used by each sector's R\&D activity are, the higher the marginal productivity of labor in R\&D is, and eventually the higher the growth rate is.

The paper tackles the apparent following paradox. Knowledge diffusion seems to lead to scale effects; however, the former is empirically desirable while the latter is not. Our first basic result is that a sufficient condition to have a scale-invariant fully endogenous growth model is to assume no inter-sectoral knowledge diffusion. However, this assumption is not empirically reasonable. We overcome the aforementioned paradox by showing that the absence of diffusion is not a necessary condition to suppress scale effects. More precisely, we determine sets of reasonable assumptions on knowledge diffusion under which one can obtain fully endogenous growth models complying with most undeniable empirical facts - namely the absence of significant scale effects, the impact of public policies on the growth rate, and somehow realistic interactions among sectors R\&D activities (including the occurrence of GPTs).
\end{abstract}

Keywords Schumpeterian growth theory / Scale effects / Inter-sectoral knowledge diffusion / Knowledge spillovers / Non rivalry

JEL Classification O30/O31/O33/O40/O41

Acknowledgements We wish to thank Philippe Aghion, Hippolyte d'Albis, Jean-Pierre Amigues, Jean-Luc Gaffard, Peter Howitt, Maurizio Iacopetta, Pietro Peretto, Gilles Saint-Paul, John J. Seater, Francesco Vona, and the participants of the Workshop on Economic Growth (2011 and 2012) organized by NC State, OFCE and SKEMA Business School for their helpful comments and suggestions.

\section{Introduction}

The seminal models of endogenous growth theory based on innovation, as initiated by Romer (1990), Grossman \& Helpman (1991), or Aghion \& Howitt (1992), all have in common to predict that the economy's long-run per capita growth rate increases in its size, measured by the population level. The presence of this scale effects property is strongly inconsistent with twentieth century

*E. Gray (corresponding author), Université de Toulouse, Toulouse Business School - Address: 20 Boulevard Lascrosses, BP 701031068 Toulouse Cedex 7, France - Tel.: +33 (0)5.61.29.49.25 - Email: e.gray@tbs-education.fr

$\dagger$ A. Grimaud, Université de Toulouse, Toulouse School of Economics and Toulouse Business School - Address: 20 Boulevard Lascrosses, BP 701031068 Toulouse Cedex 7, France - Email: andre.grimaud@tse-fr.eu 
observed stylized facts. Indeed, empirical evidence both for the United States (e.g. Backus, Kehoe \& Kehoe 1992) and for OECD countries (e.g. Jones 1995a) have invalidated the fact that, the larger the scale of the economy is, the stronger growth will be. Furthermore, these models do not allow to consider population growth: if population is assumed to grow at a positive and constant rate, the economy's per capita growth rate increases exponentially over time and eventually becomes infinite in the steady-state.

The literature generally relates the presence of scale effects in innovation-based growth models with the non rivalry property of knowledge (each unit of knowledge can be used simultaneously and infinitely by any agent in the economy without precluding its use by any other agent). As explained, for instance, by Eicher \& Turnovsky (1999) or Jones (1999, 2005), this property (often referred to as "knowledge spillovers") implies that increasing returns to scale is likely to characterize production possibilities. That is why, as the population size increases, knowledge is used by more agents, thus leading the economy to grow at a higher rate.

In the present paper, we investigate further the link between scale effects and non rivalry of knowledge. Our motivation is the following. Non rivalry is an intrinsic property of knowledge as a good in that any given unit of knowledge can indeed potentially be used by any agent, notably by the research and development $(\mathrm{R} \& \mathrm{D})$ activity of any sector. However, it does not necessarily mean that it is effectively used by all of these agents (in particular, some sectors' R\&D activity may not use it). Many empirical studies (e.g. Griliches, 1992, 1995; Hall, Mairesse \& Mohnen, 2010) have underlined interactions between sectors (i.e. inter-sectoral knowledge spillovers). Yet, as argued above, these interactions may not be global; for instance, Hall, Mairesse \& Mohnen (2010) state that "such spillovers are all the more likely and significant as the sender and the receiver are closely related". In other words, even if knowledge is a non rival good, its diffusion across sectors may be partial. As a matter of fact, several types of innovations coexist. On one end, some innovations involve specific knowledge which is used only within the sector in which this knowledge has been produced and whose productivity is nil in other sectors. On the other end, some innovations entail knowledge that is likely to impact a wide range of sectors of the economy (potentially all sectors); such innovations refer to the concept of "general-purpose technologies" (GPTs) as coined by Bresnahan \& Trajtenberg (1995), who argue that some particular innovations "are characterized by pervasiveness [...], inherent potential for technical improvements". One can cite as examples of such innovations, in ancient times, writing and printing, and in more recent times, electricity, microchip or information and communication technologies (ICTs). ${ }^{1}$ Naturally, all intermediate cases, ranging from only intra-sectoral knowledge diffusion to global knowledge diffusion, exist and should be considered.

In order to formalize these features of knowledge, we introduce explicitly inter-sectoral knowledge diffusion by using a Salop (1979) circle. $^{2}$ Then, we analyze the issue of scale effects by plugging this formalization in a standard Schumpeterian growth model. It will appear clearly that non rivalry of knowledge does not necessarily imply scale effects and that, if these effects occur, they basically result from a sufficiently broad diffusion of knowledge.

The issue of scale effects has been reviewed in a large body of literature in which it is commonly agreed that this non desirable property is fundamentally linked to the technologies considered in the models, in particular to the production function of innovations. More precisely, the process of innovation is based on that $R \& D$ produces knowledge using two types of inputs: rival ones (e.g. labor, capital) and a stock of knowledge. The scale effects issue is thus basically linked to the way these inputs are taken into account by the formalization of this knowledge production function.

\footnotetext{
${ }^{1}$ For more details on the theory and applications of GPTs, we refer the reader to the essays included in Helpman (1998), or to Aghion \& Howitt (2009, Ch.9).

${ }^{2}$ The term "diffusion" has also been used to refer to the phenomenon involving that "there is a lag between the appearance of a technology and its peak usage" (Chari \& Hopenhayn 1991). The link between this temporal dimension of knowledge diffusion and scale effects has been studied for instance by Schulstad (1993); he shows that "the introduction of a diffusion process through which new technology is gradually incorporated into the economywide stock of knowledge can reduce and eliminate the dependence of the growth rate upon the size of the economy". In this paper, we abstract away from this temporal dimension of knowledge diffusion by considering instantaneous diffusion, and we focus on the link between "spatial diffusion" and scale effects. The introduction of a lag involved by technology adoption remains to be explored within our model and is left for further research (one could for instance consider that the more distant two sectors are, the longer the lag in technology adoption).
} 
Consequently, a variety of models has eliminated scale effects by modifying the technologies initially introduced in endogenous growth models. This has been explained, for instance, in Jones (1999, 2005), in Li $(2000,2002)$, in Laincz \& Peretto (2006), in Dinopoulos \& Sener (2007), or in Ha \& Howitt (2007). These papers have identified two major ranges of models suppressing scale effects. ${ }^{3}$

A first range of models, including Jones (1995b), Kortum (1997), or Segerstrom (1998), based on the notion of "diminishing technological opportunities", has given birth to the "semi-endogenous growth" literature. This approach provides scale-invariant growth models, in which the long-run growth rate of the economy is proportional to the exogenous rate of population growth. More precisely, these models exhibit "weak scale effects": scale effects are still present in the determination of the variables levels but no longer of their growth rates. ${ }^{4}$ Moreover, in the absence of positive population growth, the growth rate of the economy is nil. Finally, economic policies - especially subsidies to R\&D - turn to have an impact only on the levels of economic variables, not on the long-run growth rates. In the semi-endogenous growth models, returns to scale are still increasing, and (strong) scale effects are suppressed by assuming decreasing returns in the stock of knowledge.

Contrasting with the semi-endogenous growth theory, an alternative range of literature - that one often refers to as "(fully) endogenous growth without scale effects" theory - appeared through the impulse of Aghion \& Howitt (1998), Dinopoulos \& Thompson (1998), Peretto (1998), Young (1998), Howitt (1999), Peretto \& Smulders (2002), among others. This second approach restores the effect of economic policies on long-term growth, without displaying the scale effects property. Contrary to semi-endogenous growth models, scale-invariant fully endogenous growth models consider constant returns in the stock of knowledge. Scale effects are eliminated through a "variety expansion mechanism". This mechanism is in line with Young (1998)'s insight that, as population grows, the proliferation of sectors reduces the efficiency of $R \& D$ activities in improving the quality of an existing product because the R\&D effort is diluted in more sectors. As explained by Dinopoulos \& Sener (2007), "horizontal product differentiation takes the form of variety accumulation and removes the scale effects property from these models [...]. Vertical product differentiation takes the form of quality improvements or process innovations and generates endogenous long-run growth". As argued in Jones (1999, 2005), Peretto \& Smulders (2002), Laincz \& Peretto (2006), Dinopoulos \& Sener (2007), Ha \& Howitt (2007), or Aghion \& Howitt (2009, Ch.4), scale effects are eliminated by means of two assumptions introduced in the canonical endogenous growth models. The first one consists in assuming that the scale of the economy, as measured by the level of its population, has an impact on the number of sectors (i.e. the variety of goods). The second one relates to the formalization of the process of knowledge accumulation. Analyzing how scale effects are suppressed via these two assumptions enables us to understand the sources of scale effects.

The first possible source of scale effects is the following. As the size of the population increases, so does the quantity of labor devoted to $\mathrm{R} \& \mathrm{D}$ in each sector (i.e. the $\mathrm{R} \& \mathrm{D}$ effort per sector). Consequently, each sector produces more innovations, which fosters growth. The assumption along which the level of the population impacts the number of sectors ${ }^{5}$ offsets this source of scale effects: as the population size increases, sectors proliferation dilutes $R \& D$ effort in a larger number of different sectors, thus dissipating its effect on the overall rate of productivity growth.

The second source of scale effects is more intricate as it relates to the nature of knowledge spillovers. One can summarize it as follows. Given that an increase in the population level implies an increase in the number of sectors, if the knowledge produced in each sector diffuses to a large set of sectors, each of these sectors would thus have access to more knowledge and would hence

\footnotetext{
${ }^{3}$ In these papers, one finds quite exhaustive surveys of the literature on scale effects, as well as synthetic expositions of the various functional forms of knowledge production adopted to suppress them. One can also find approaches focussing on the final good production function, as for instance in Aghion \& Howitt (2009, Ch. 4, see equation 4.17).

${ }^{4}$ Two forms of scale effects can be identified in the literature: "strong scale effects", as in the first generation of endogenous growth models, and "weak scale effects" as in the semi-endogenous growth models. For more details on this distinction, see, for instance, Jones (2005). In the present paper, like in most of the literature tackling this issue, we focus on "strong scale effects" to which one generally refers to as "scale effects". From now on, we use the same language abuse.

${ }^{5}$ This assumption has been widely debated by the related literature and a consensus has been reached: most models consider that an increase in population size results in a proportionate increase in the number of sectors (e.g. Peretto 1998; Young 1998; Aghion \& Howitt 1998, Ch. 12; Dinopoulos \& Thompson 1998; Howitt 1999). This feature, that can be derived from market-based mechanisms (see, for instance, Dinopoulos \& Sener 2007 for more details), has also been justified empirically (see, for instance, Laincz \& Peretto 2006). We return on this point in Subsection 2.1 - Assumption 1, when we introduce formally this proportionality assumption.
} 
produce more innovations. In short, if knowledge spillovers are sufficiently large, an increase in the population level can lead to a higher growth rate. As it will be argued in this paper, the second assumption introduced to eliminate scale effects somehow consists in suppressing inter-sectoral knowledge spillovers.

The model developed in this paper is in line with the fully endogenous Schumpeterian growth theory. ${ }^{6}$ We use the commonly agreed assumption along which an increase in the population size results in a proportionate increase in the number of sector. Then, our main focus is to analyze in detail the link between knowledge diffusion (i.e. knowledge spillovers) and scale effects.

The starting point of our analysis is the following. As recalled above, R\&D activity produces knowledge (innovations) using two types of inputs: a rival one (labor) and a non rival one. This non rival input consists in a given stock of knowledge, that we will name "pool of knowledge". When introducing this type of knowledge production function within a simple Schumpeterian growth model, one basically obtains the following enlightening result. On the one hand, if the pool of knowledge used by R\&D activity in each sector is the largest possible pool (all the knowledge accumulated so far in the economy) - that is, if one assumes knowledge spillovers across all sectors - then, the model exhibits scale effects. On the other hand, if the pool of knowledge used by each sector's R\&D activity comprises only the knowledge accumulated so far in this sector - that is, there are no inter-sectoral knowledge spillovers - then, there are no scale effects. Why is it so?

In fact, because these two inputs are complements, the size of the pool of knowledge used in each sector is obviously a key determinant of the marginal productivity of labor in this sector's R\&D activity. As a matter of example, let us return to the two previous polar cases. In the case of generalized inter-sectoral knowledge spillovers, as the size of the economy (i.e. population level) increases, the size of the pool used in each sector - and thus the marginal productivity of labor in R\&D - increases. This generates scale effects. On the contrary, in the case without any inter-sectoral knowledge spillovers, the size of the pool used in each sector - and thus the marginal productivity of labor in $R \& D$ - are independent of the size of the population. That is why there are no scale effects.

The first key point in understanding the issue of scale effect is thus to determine the size of the pool of knowledge used in each sector to produce its own knowledge. The second key point lies in that this size depends directly on the intensity of knowledge spillovers, that is on whether knowledge diffuses across sectors and, if so, on the scope of this diffusion. Therefore, in order to study the link between scale effects and knowledge diffusion, it is necessary to provide a specific formalization that introduces explicitly inter-sectoral knowledge diffusion, and thus that explains the formation of pools. For that purpose, we exploit the circular product differentiation model of Salop (1979): each sector is located on the Salop circle and knowledge can diffuse more or less over the circle. This formalization encompasses the two polar cases mentioned above. In the case of generalized inter-sectoral knowledge spillovers (in which the pools are the largest), the knowledge produced in each sector diffuses over the whole circle (i.e. diffuses across all sectors). Thus, the R\&D activity of each sector uses the knowledge accumulated in this sector so far as well as the knowledge accumulated in any other sector. In the case without inter-sectoral knowledge spillovers, the knowledge produced in each sector does not diffuse over the circle: the pool of knowledge used by the R\&D activity of each sector comprises only the knowledge of this sector. As it will be explained below, this formalization also allows us to consider any case in between and, more generally, to consider simultaneously different types of knowledge diffusion.

Basically, we face the following paradox. On the one hand, we have seen that scale effects can be suppressed by eliminating inter-sectoral knowledge diffusion. But the absence of these inter-sectoral knowledge spillovers is not empirically reasonable. Indeed, the significance of the interactions between sectors resulting from this non rivalry has been emphasized both theoretically and empirically (e.g. Griliches, 1992, 1995; Aghion \& Howitt 1998; Hall, Mairesse \& Mohnen, 2010). On the other hand, we have also seen that the literature has underlined the fact that there

\footnotetext{
${ }^{6}$ Successive reviews have given rise to a debate regarding the respective relevancy of using semi-endogenous or fully endogenous models. Li (2000), for instance, argues that the former methodology is more general than the latter. Ha \& Howitt (2007) maintain that fully endogenous growth is more accurate; like Madsen (2008), they argue that empirical evidences are more supportive of fully endogenous Schumpeterian growth theory than they are of semi-endogenous growth theory.
} 
is no evidence of significant scale effects. This leads us to the main question of this paper: is it possible to develop a fully endogenous Schumpeterian growth model that maintains inter-sectoral knowledge diffusion while remaining in accordance with the empirical facts regarding scale effects? More precisely, does it exist reasonable assumptions on knowledge diffusion such that the results given by the model are relevant regarding both the scale effects property and this diffusion?

To tackle this paradox, we use the following three steps methodology. First, we provide a formalization that encompasses simultaneously various types of knowledge diffusion across the set of sectors of the Salop circle. More precisely, we consider that some sectors produce knowledge that diffuses across the whole set of sectors, some sectors produce knowledge that does not diffuse to any other one, and finally some sectors produce knowledge that diffuses toward a more or less wide set of sectors. The type of knowledge diffusion considered in the first range of sectors echoes to the concept of GPTs mentioned above. The type of knowledge diffusion considered in the second range of sectors has often been used in the fully endogenous growth theory without scale effects (e.g. Segerstrom 1998; Peretto 1999; Acemoglu 2009, Ch. 14; or Aghion \& Howitt 2009, Ch. 4). The third range of sectors allows us to consider any intermediate type of knowledge diffusion. This formalization enables us to explain how the pools of knowledge arise. Second, we use the commonly agreed assumption of stochastic arrival of innovations in each sector, which depends on the level of R\&D effort (labor devoted to R\&D). Furthermore, we introduce the new assumption along which, in each sector, consequently to each innovation, the jump on the quality ladder (i.e. the increase in knowledge) depends explicitly on the pool of knowledge in which this sector's R\&D draws from. Third, we plug the law of knowledge accumulation thereby derived in a standard Schumpeterian endogenous growth model with vertical innovations (the two polar cases mentioned above are in fact two particular cases of this model).

This fully endogenous growth model allows us to revisit the issue of scale effects under the new light of knowledge diffusion. In this general framework, we confirm the simple intuition presented above: scale effects depend on knowledge diffusion because diffusion determines the size of the pools, and thus the productivity of labor in $\mathrm{R} \& \mathrm{D}$. Then, we get the following three main results.

i) The first basic result we obtain is that, in the absence of any inter-sectoral knowledge diffusion (in particular no GPTs), the model does not exhibit scale effects (in fact, we show that this is what has been assumed explicitly or implicitly in most scale-invariant fully endogenous growth models so far).

ii) We investigate whether assuming no inter-sectoral knowledge diffusion (assumption which obviously appears as counterfactual) is required to have a scale-invariant fully endogenous growth model. We show that it is a sufficient but not necessary condition to suppress scale effects. Indeed, we provide a set of assumptions on knowledge diffusion such that there are no scale effects, public policies have an impact on the growth rate, and there still remains some basic inter-sectoral knowledge diffusion. These assumptions may however appear questionable insofar as they exclude the possible arrival of GPTs knowledge and they impose some restrictions on the way knowledge diffuses (as the size of the economy increases, the scope of diffusion remains unchanged). These basic assumptions are then relaxed as explained in iii) below.

iii) In order to comply with most of the commonly agreed empirical facts regarding growth models - namely the absence of significant scale effects, the effects of public policies, and somehow realistic interactions among sectors $R \& D$ activities (including the occurrence of GPTs) - we relax the two assumptions used in ii). First, assuming possible arrival of GPTs implies the presence of scale effects; however, we show that their significance is weak because it depends on the probability of occurrence of GPTs which is obviously low. Second, regarding the assumption along which the scope of knowledge diffusion may be impacted by the size of the economy, we derive the following results. If it is assumed that this scope expands with the size of the economy, there are scale effects but, under some reasonable extra assumptions, their significance decreases with this size and asymptotically vanishes. If it is assumed that the scope of knowledge diffusion contracts as the size of the economy increases, there are negative scale effects. To sum up, we manage to provide a Schumpeterian endogenous growth model in accordance with the main empirical facts related to innovation-based growth theory.

The paper is organized as follows. The basic intuitions regarding the link between scale effects and knowledge diffusion are presented in Section 2. In Section 3, we develop a Schumpeterian growth model with explicit knowledge diffusion. In Section 4, we use this model to revisit the issue 
of scale effects. We conclude in Section 5. All computations are provided in Appendix - Section 6.

\section{Scale effects and knowledge diffusion: basic intuitions}

In this section, in order to present the main intuitions on the link between knowledge diffusion and scale effects, we use a simplified version of the fully endogenous growth Schumpeterian model that will be exhaustively developed in Section 3 and used to revisit the issue of scale effects in Section 4. In particular, we show that this link goes through the following channel. Schematically, if inter-sectoral knowledge diffusion is narrow (resp. wide), an increase in the size of the economy (i.e. of the population) leads to a low (resp. high) increase in the marginal productivity of labor in R\&D activity, and thus to the absence (resp. presence) of scale effects. This channel will be analyzed in detail in Section 4.

\subsection{Basic assumptions}

There is a continuum $\Omega_{t}$, of measure $N_{t}$, of intermediate sectors uniformly distributed on a clockwise oriented circle in the spirit of Salop (1979). At each date $t$, each sector $\omega, \omega \in \Omega_{t}$, is characterized by a stock of knowledge $\chi_{\omega t}$ and by an intermediate good $\omega$, produced in quantity $x_{\omega t}$, which embodies this stock of knowledge. As usual in endogenous growth theory, we assume that all sectors have an identical initial level of knowledge: $\chi_{\omega 0}=\chi_{0}, \forall \omega \in \Omega_{t}{ }^{7}$ Assuming that knowledge is homogenous, the whole stock of knowledge in the economy at each date $t$ is

$$
\mathcal{K}_{t}=\int_{\Omega_{t}} \chi_{\omega t} d \omega
$$

The initial stock $\mathcal{K}_{0}$ is normalized to one.

The model developed in this paper is in direct line with the endogenous growth models without scale-effects that allow for constant population growth while maintaining the effects of public policies. In these fully endogenous growth models, scale effects are removed through a "variety expansion mechanism". More precisely, as underlined by Jones (1999), Laincz \& Peretto (2006), Dinopoulos \& Sener (2007), or Aghion \& Howitt (2009, Ch. 4), these models all have in common to somehow assume that the number of sectors $N_{t}$ increases as the population level $L_{t}$ does so. In what follows, we will refer to $L_{t}$ or indifferently $N_{t}$ as to the "size of the economy". Formally, we make the following standard assumption. ${ }^{8}$

Assumption 1. The number of sectors $N_{t}$ and the size of the population $L_{t}$ are proportional: $N_{t}=\gamma L_{t}, \gamma>0$. Population grows at constant rate $g_{L_{t}}=n, n \geqslant 0$, and its initial size, $L_{0}$, is normalized to one.

\footnotetext{
${ }^{7}$ We provide more detail on the assumption of symmetry across sectors - which is standard in endogenous growth theory - below (see 2.2, footnote 11).

${ }^{8}$ There are several ways to justify a linear relation between the number of sectors and the population level. The main argument put forward by Laincz \& Peretto (2006) is empirical. They show that, even though this relation "might induce one to conclude that this class of models requires another 'knife-edge' condition in that one needs to assume that the number of firms is exactly proportional to population", the number of establishments is indeed proportional to employment according to their data. Furthermore, this relation can also be justified theoretically. As stated by Dinopoulos \& Sener (2007), "the linear relationship between the number of varieties and the level of population can be derived from market-based mechanisms with solid micro foundations." For instance, in Young (1998, see equation 17), it is derived under the standard assumptions of fixed-entry costs and monopolistic competition. In Howitt (1999) and in Segerstrom (2000), it is introduced endogenously. In Aghion \& Howitt (1998, Ch. 12 , and 2009 , Ch. 4 ,), it is set up in a more straightforward scheme which could be adapted here to obtain the linear relation of Assumption 1: assume that the probability of inventing a new intermediate good at date $t$ is a linear function of the population size and that, at each date $t$, an exogenous fraction of intermediate goods becomes obsolete and vanishes. Then, the variation of the number of sectors at date $t$ is given by $\dot{N}_{t}=\kappa L_{t}-\xi N_{t}$, where $\kappa$ and $\xi$ are positive parameters, and where $L_{t}=e^{n t}$. The solution of this differential equation is $N_{t}=\frac{\kappa}{n+\xi}\left(e^{n t}-e^{-\xi t}\right), \forall t$; dividing both sides by $L_{t}$ gives $\frac{N_{t}}{L_{t}}=\frac{\kappa}{n+\xi} \frac{e^{n t}-e^{-\xi t}}{e^{n t}}=\frac{\kappa}{n+\xi}\left(1-e^{-(\xi+n) t}\right), \forall t$. Consequently, since $\xi+n>0$, the ratio number of intermediate sectors over population level will eventually stabilize at a steady-state value, $\left(N_{t} / L_{t}\right)^{s s}=\kappa /(n+\xi) \equiv \gamma$.

Note that, Jones (1999) provides a discussion on the more general relation $N_{t}=L_{t}^{\beta}, \beta \gtreqless 1$. Our analysis could be generalized under this more general assumption.
} 
It is commonly agreed that new knowledge is produced using two types of inputs: rival goods (e.g. labor, physical capital, final good) and a non rival good (a stock of knowledge previously created). The mechanism at the source of the creation of knowledge - that is the law of knowledge accumulation - relies on two core assumptions.

Firstly, we assume stochastic arrival of innovations as initially introduced in Grossman \& Helpman (1991) or in Aghion \& Howitt (1992):

Assumption 2. If $l_{\omega t}$ is the amount of labor devoted to RED at date $t$ in any intermediate sector $\omega, \omega \in \Omega_{t}$, to move on to the next quality of intermediate good $\omega$, innovations occur randomly with a Poisson arrival rate $\lambda l_{\omega t}, \lambda>0$.

Secondly, each R\&D activity creates new knowledge making use of previously created knowledge. This idea is formalized by considering that, in order to produce new knowledge, in each sector $\omega, \mathrm{R} \& \mathrm{D}$ activity draws from a specific pool of knowledge $\mathcal{P}_{\omega t}$. Formally, we assume that, for any intermediate good $\omega, \omega \in \Omega_{t}$, if an innovation occurs at date $t$, the increase in knowledge $\Delta \chi_{\omega t}$ (i.e. the quality improvement of the intermediate good) depends positively on the current size of this pool of knowledge:

Assumption 3. For any intermediate sector $\omega, \omega \in \Omega_{t}$, if an innovation occurs at date $t$, the increase in knowledge is $\Delta \chi_{\omega t}=\sigma \mathcal{P}_{\omega t}, \sigma>0$.

In order to simplify computations, only the input $l_{\omega t}$ (resp. the input $\left.\mathcal{P}_{\omega t}\right)$ appears in Assumption 2 (resp. Assumption 3); moreover, the relations considered in these two assumptions are assumed to be linear. From Assumptions 2 and 3, one derives the law of motion of the average knowledge inherent in any sector $\omega:^{9}$

Proposition 1. Under Assumptions 2 and 3, the expected knowledge in any intermediate sector $\omega, \omega \in \Omega_{t}$, is a differentiable function of time. The law of knowledge accumulation is 10

$$
\dot{\chi}_{\omega t}=\lambda \sigma l_{\omega t} \mathcal{P}_{\omega t}, \forall \omega \in \Omega_{t}
$$

\section{Proof. See Appendix 6.1}

The law of knowledge accumulation resulting from Assumptions 2 and 3 has properties that are standard in endogenous growth theory. It is a Cobb-Douglas production function with constant returns in the rival input $l_{\omega t}$, and increasing-returns-to-scale in $l_{\omega t}$ and $\mathcal{P}_{\omega t}$ taken together, since the later is a non rival input. In each sector $\omega$, the marginal productivity of labor in the production of knowledge is an increasing linear function of the pool of knowledge $\mathcal{P}_{\omega t}$ used by R\&D activity in this sector:

$$
\frac{\partial \dot{\chi}_{\omega t}}{\partial l_{\omega t}}=\lambda \sigma \mathcal{P}_{\omega t}, \forall \omega \in \Omega_{t}
$$

In all what follows, $g_{z_{t}}$ denotes the rate of growth, $\dot{z}_{t} / z_{t}$, of any variable $z_{t}$. From (2) and (3), one gets

$$
\dot{\chi}_{\omega t}=l_{\omega t} \frac{\partial \dot{\chi}_{\omega t}}{\partial l_{\omega t}} \text { and thus } g_{\chi_{\omega t}}=\frac{l_{\omega t}}{\chi_{\omega t}} \frac{\partial \dot{\chi}_{\omega t}}{\partial l_{\omega t}}, \forall \omega \in \Omega_{t}
$$

\footnotetext{
${ }^{9}$ More generally, one could assume that the Poisson arrival rate in Assumption 2 is a function $\lambda\left(l_{\omega t}, \mathcal{P}_{\omega t}\right)$ and that the increase in knowledge, $\Delta \chi_{\omega t}$, in Assumption 3 is a function $\sigma\left(l_{\omega t}, \mathcal{P}_{\omega t}\right)$. Accordingly, one would obtain the following law of knowledge accumulation: $\dot{\chi}_{\omega t}=\lambda\left(l_{\omega t}, \mathcal{P}_{\omega t}\right) \sigma\left(l_{\omega t}, \mathcal{P}_{\omega t}\right), \forall \omega \in \Omega_{t}$. This law is rather general and encompasses most of the ones used in the literature. For instance, if one assumes $\lambda\left(l_{\omega t}, \mathcal{P}_{\omega t}\right)=\lambda l_{\omega t}($ as in Assumption 2), $\sigma\left(l_{\omega t}, \mathcal{P}_{\omega t}\right)=\sigma \mathcal{P}_{\omega t}$ (as in Assumption 3), and furthermore $\mathcal{P}_{\omega t}=\chi_{\omega t}$, one gets a law which is similar to the ones considered in Grossman \& Helpman (1991), Segerstrom (1998), Peretto (1999), Acemoglu (2009, Ch. 14), or Aghion \& Howitt (2009, Ch. 4): $\dot{\chi}_{\omega t}=\lambda \sigma l_{\omega t} \chi \omega t, \forall \omega \in \Omega_{t}$.

In the same way, assuming $\lambda\left(l_{\omega t}, \mathcal{P}_{\omega t}\right)=\lambda l_{\omega t}, \sigma\left(l_{\omega t}, \mathcal{P}_{\omega t}\right)=\sigma \mathcal{P}_{\omega t}$, and $\mathcal{P}_{\omega t}=\chi_{t}^{\max } \equiv \max \left\{\chi_{\omega t}, \omega \in \Omega_{t}\right\}$, one gets $\dot{\chi}_{\omega t}=\lambda \sigma l_{\omega t} \chi_{t}^{\max }, \forall \omega \in \Omega_{t}$, a law which is similar to the one initially introduced in Aghion \& Howitt (1992).

A last example is the following. Assuming $\lambda\left(l_{\omega t}\right)=\lambda l_{\omega t}(\lambda>0), \sigma\left(\mathcal{P}_{\omega t}\right)=\sigma \mathcal{P}_{\omega t} \Phi(\sigma>0$ and $\Phi<1)$ and $\mathcal{P}_{\omega t}=\mathcal{K}_{t}$, one gets $\dot{\chi}_{\omega t}=\lambda \sigma l_{\omega t} \mathcal{K}_{t}{ }^{\Phi}, \forall \omega \in \Omega_{t}$. Furthermore, assuming $N_{t}=N$ (i.e. $\left.\Omega_{t}=\Omega\right)$ and summing on $\Omega$, one obtains $\dot{\mathcal{K}}_{t}=\lambda \sigma L_{t}^{R} \mathcal{K}_{t} \Phi$, where $L_{t}^{R}$ denotes the overall amount of labor dedicated to R\&D activities in the economy: $L_{t}^{R}=\int_{\Omega} l_{\omega t} d \omega$. This law of knowledge accumulation is formally identical to those assumed in the semi-endogenous growth theory. Indeed, this theory is presented using a similar expression in Jones (1999, equation 4), in Laincz \& Peretto (2006, equation 5), in Dinopoulos \& Sener (2007, equations 2 and 6), in Ha \& Howitt (2007, equation 3), or in Acemoglu (2009, Ch. 13, equation 13.34).

${ }^{10}$ The expectation operator is dropped to simplify notations: $\frac{\partial \mathbb{E}\left[\chi_{\omega t}\right]}{\partial t} \equiv \dot{\chi}_{\omega t}$.
} 
Finally, it remains to explain in what consists exactly these pools of knowledge and how they are shaped. In Subsection 3.1 below, we provide a formalization that allows us to understand how the pools $\mathcal{P}_{\omega t}$ are formed. In particular, we will explain how knowledge can diffuse on a more or less large subset of sectors located on the Salop circle (possibly the whole circle): it will be made clear that the broader the knowledge diffusion over the circle is, the larger the pools are. There are two polar cases:

i) The case in which the knowledge produced in any sector diffuses across the whole set of sectors $\Omega_{t}$ (we will refer to this type of knowledge diffusion as to "global inter-sectoral knowledge diffusion"). Here, all the pools of knowledge are identical and comprise the whole disposable knowledge in the economy: $\mathcal{P}_{\omega t}=\mathcal{K}_{t}, \forall \omega \in \Omega_{t}$.

ii) The case in which there is no inter-sectoral knowledge diffusion over the circle. Here, each sector $\omega, \omega \in \Omega_{t}$, uses only its own knowledge. Thus, its pool of knowledge is $\mathcal{P}_{\omega t}=\chi_{\omega t}$.

We can now present some results providing the basic intuitions revealing the link between knowledge diffusion and the scale effect property.

\subsection{Basic intuitive results}

In order to get these results as directly as possible, let us use some standard assumptions and properties that are usual in growth models and that will be detailed and proved in the subsequent sections. First, we consider the standard assumption of symmetry across sectors. ${ }^{11}$ The quantity of labor devoted to R\&D in all sectors is assumed to be the same: $l_{\omega t}=l_{t}, \forall \omega \in \Omega_{t}$. Similarly, the level of knowledge characterizing each sector and the pool of knowledge used by its R\&D activity are both also independent of $\omega: \chi_{\omega t}=\chi_{t}$ and $\mathcal{P}_{\omega t}=\mathcal{P}_{t}, \forall \omega \in \Omega_{t}$. Second, when computing the Schumpeterian equilibrium, we will prove the two following results: the growth rate of per capita consumption is given by $g_{c_{t}}=g_{\chi_{t}}+n$, and $l_{t}$ is independent of $L_{t}$ (see Proposition 4 and its proof).

As mentioned above, the main purpose of this paper is to exhibit the fundamental link between knowledge diffusion and scale effects. Recall that tackling the issue of scale effects consists in studying the impact of the size of the economy $L_{t}$ on the growth rate of per capita consumption $g_{c_{t}}$. Using (4), one expresses $g_{c_{t}}$ as a function of the marginal productivity of labor in R\&D $\frac{\partial \dot{\chi} t}{\partial l_{t}}$, which itself depends on the pool of knowledge $\mathcal{P}_{t}$ :

$$
g_{c_{t}}=g_{\chi_{t}}+n=l_{t} \frac{\partial \dot{\chi}_{t} / \partial l_{t}}{\chi_{t}}+n, \text { where } \frac{\partial \dot{\chi}_{t}}{\partial l_{t}}=\lambda \sigma \mathcal{P}_{t}
$$

As shown in (5), studying the issue of scale effects thus reduces to examining the impact of $L_{t}$ on the growth rate of knowledge in each sector $g_{\chi_{t}}$, hence on the ratio $\frac{\partial \dot{\chi}_{t} / \partial l_{t}}{\chi_{t}}$, therefore on the marginal productivity of labor in each sector R\&D activity, and thus finally on the pools of knowledge which result from knowledge diffusion. Considering the two polar cases described above, the following proposition provides a simple illustration showing that knowledge diffusion has a direct impact on the presence of scale effects.

Proposition 2. The link between knowledge diffusion and scale effects is illustrated in the two following polar cases:

i) If the knowledge produced in any sector $\omega$ diffuses across the whole set of sectors $\Omega_{t}$ (i.e. $\left.\mathcal{P}_{\omega t}=\mathcal{K}_{t}, \forall \omega \in \Omega_{t}\right)$, there are scale effects.

ii) If there is no inter-sectoral knowledge diffusion (i.e. $\mathcal{P}_{\omega t}=\chi_{\omega t}, \forall \omega \in \Omega_{t}$ ), there are no scale effects.

\footnotetext{
${ }^{11}$ The assumption of symmetry across sectors is commonly made in endogenous growth theory; see, for instance, Aghion \& Howitt (1992 or 1998 - Ch. 3), or Peretto \& Smulders (2002). For more details on this issue, the reader can refer to Peretto $(1998,1999)$ or to Cozzi, Giordani \& Zamparelli (2007) in which the relevancy of the symmetric equilibrium is discussed.
} 
Proof.

i) Assume that $\mathcal{P}_{\omega t}=\mathcal{K}_{t}, \forall \omega \in \Omega_{t}$. Under Assumption 1 and the symmetry assumption, (1) writes $\mathcal{K}_{t}=N_{t} \chi_{t}=\gamma L_{t} \chi_{t}, \forall \omega \in \Omega_{t}$. Accordingly, one has $\mathcal{P}_{\omega t}=\mathcal{P}_{t}=\gamma L_{t} \chi_{t}, \forall \omega \in \Omega_{t}$. Consequently, in this polar case with "global inter-sectoral knowledge diffusion", one has $\frac{\partial \dot{\chi}_{\omega t}}{\partial l_{\omega t}}=\frac{\partial \dot{\chi}_{t}}{\partial l_{t}}=\lambda \sigma \mathcal{P}_{t}=\lambda \sigma \gamma L_{t} \chi_{t}, \forall \omega \in \Omega_{t}$. Finally, from (5) one gets $g_{c_{t}}=l_{t} \frac{\partial \dot{\chi}_{t} / \partial l_{t}}{\chi_{t}}+n=$ $l_{t} \frac{\lambda \sigma \mathcal{P}_{t}}{\chi_{t}}+n=l_{t} \lambda \sigma \gamma L_{t}+n$. Since $l_{t}$ is independent of $L_{t}$, there are scale effects.

ii) Assume that $\mathcal{P}_{\omega t}=\chi_{\omega t}, \forall \omega \in \Omega_{t}$. Under the symmetry assumption, $\mathcal{P}_{\omega t}=\mathcal{P}_{t}=\chi_{t}, \forall \omega \in \Omega_{t}$. Hence, in this polar case without any inter-sectoral knowledge diffusion, one has $\frac{\partial \dot{\chi}_{\omega t}}{\partial l_{\omega t}}=$ $\frac{\partial \dot{\chi}_{t}}{\partial l_{t}}=\lambda \sigma \mathcal{P}_{t}=\lambda \sigma \chi_{t}, \forall \omega \in \Omega_{t}$. Finally, from (5) one obtains $g_{c_{t}}=l_{t} \frac{\lambda \sigma \mathcal{P}_{t}}{\chi_{t}}+n=l_{t} \lambda \sigma+n$. Here also, $l_{t}$ is independent of $L_{t}$; thus there are no scale effects.

The main intuitions regarding Proposition 2 are as follows. We have seen above that the presence of scale effects - that is the impact of the size of the economy $L_{t}$ on the growth rate $g_{c_{t}}$ - is basically related to the size of the pools, and thus to the magnitude of knowledge diffusion across the sectors' R\&D activities. How can the underlying mechanism at the origin of the link between scale effects and knowledge diffusion be understood?

As the size of the population $L_{t}$ increases, the number of sectors $N_{t}$ increases as well (see Assumption 1). If inter-sectoral knowledge diffusion is maximum (any innovation diffuses to the whole set of sectors as in the polar case $i)$ ), the size of the pools of knowledge increases with the size of the economy (as measured by $L_{t}$ or $N_{t}$ ); hence so do the marginal productivity of labor in R\&D activity $\left(\frac{\partial \dot{\chi}_{t}}{\partial l_{t}}\right)$, the growth rate of knowledge $g_{\chi_{t}}$, and eventually the growth rate of per capita consumption $g_{c_{t}}$ : there are scale effects. If there is no inter-sectoral knowledge diffusion (as in the polar case ii)), the pool used in each sector comprises solely the knowledge inherent in this sector; it is thus independent of the size of the economy. Consequently, the marginal productivity of labor in R\&D activity, the growth rate of knowledge $g_{\chi_{t}}$, and finally the growth rate of per capita consumption $g_{c_{t}}$, are not impacted by $L_{t}$ : there is no scale effect.

This analysis has underlined the key part played by knowledge diffusion in the understanding of the scale effect property. In particular, we have shown in Proposition 2-ii) that suppressing inter-sectoral knowledge diffusion is a sufficient condition to remove scale effects. Is it a necessary condition?

\section{A Schumpeterian growth model with explicit knowledge diffusion}

In order to study the questions raised in Section 2 and more generally to revisit the issue of scale effects under the light of knowledge diffusion, we develop a general Schumpeterian growth framework which considers explicitly knowledge diffusion and which encompasses the two polar cases described above as well as all intermediary cases. Fundamentally, we formalize how knowledge diffusion shapes the pools of knowledge in which $R \& D$ activities draw from to produce new knowledge.

\subsection{Knowledge diffusion and pools of knowledge}

In Section 2, we have described the knowledge accumulation process (see Proposition 1) without providing any particular specification to the pool of knowledge $\mathcal{P}_{\omega t}$ used by R\&D activity in each sector $\omega$. Now, we propose a mechanism formalizing how the constitution of each of these pools relies on the influence that $R \& D$ activities have on each other. In that respect, we explicitly introduce a process of knowledge diffusion over the Salop circle. Many empirical studies underline the fact that R\&D performed in one sector may produce positive spillovers effects on other sectors (e.g. Griliches 1992; Griliches 1995; Hall, Mairesse \& Mohnen 2010); as stated by Hall et al., "such spillovers are all the more likely and significant as the sender and the receiver are closely related". Accordingly, we consider that each sector $\omega$ on the Salop circle $\Omega_{t}$ is simultaneously a potential sender and a potential receiver of knowledge. More precisely, consider any sector $\omega, \omega \in \Omega_{t}$. As a 
sender, this sector's R\&D activity produces knowledge $\chi_{\omega t}$ which diffuses toward other sectors. As a receiver, this sector's R\&D activity uses a pool of knowledge $\mathcal{P}_{\omega t}$ which comprises the knowledge produced in this sector so far, $\chi_{\omega t}$, and potentially knowledge diffused from other sectors.

The purpose of Subsection 3.1 is twofold. First, we want to introduce a formalization that allows us to encompass various types of knowledge diffusion, ranging from no inter-sectoral diffusion to general purpose technologies (namely, any intermediate case in between the two polar cases mentioned in Section 2). Second, we want to provide an explicit expression of the pools of knowledge used by R\&D activities; obviously, this expression will depend on the type of knowledge diffusion considered. This will enable us to understand to which extent a given sector $\omega$ receives knowledge from other sectors, and eventually to determine the contribution of each sector to the pool used in a given sector $\omega$.

\subsubsection{Sending knowledge and knowledge diffusion over the Salop circle: the scope of knowledge diffusion}

Here, we aim to provide a formalization allowing us to consider simultaneously several types of knowledge diffusion. More precisely, we want to present a model with the two fundamental following features.

First, at the same date $t, i)$ some sectors produce knowledge that diffuses across the whole set of sectors, ii) some sectors produce knowledge that does not diffuse to any other one, and finally iii) some sectors produce knowledge that diffuses toward a more or less wide set of sectors on the Salop circle.

Second, we want the weights of the three types of sectors to be able to vary between zero and one, such that all cases (in fact, an infinity) comprised between the two polar cases may be encompassed. Formally, we consider the following framework.

i) A proportion $p_{G}$ of sectors are such that the knowledge produced in each of these sectors reaches all the sectors in the economy (i.e. diffuses across the whole set of sectors). We therefore consider the possible arrival of a type of knowledge diffusion that echoes to the concept of "general-purpose technologies" (GPTs) as coined by Bresnahan \& Trajtenberg (1995).

ii) A proportion $p_{s}$ of sectors are such that the knowledge produced in each of them diffuses only within this sector. In this case without any inter-sectoral knowledge diffusion, we will refer to "sector specific knowledge".

iii) A proportion $p_{m}$ of sectors are such that the knowledge they produce diffuses over a more or less wide set of sectors. The measure of this subset is denoted by $\theta_{t}, \theta_{t} \in\left(1 ; N_{t}\right)$. In this intermediate case, we will refer to "medium knowledge". We make some additional assumption on this type of knowledge.

To simplify the analysis, we assume that if a sector $\omega$ produces medium knowledge, the knowledge $\chi_{\omega t}$ diffuses symmetrically over the circle $\Omega_{t}$ from the location of sector $\omega$. Hence it diffuses on the subset of sectors $\left[\omega-\theta_{t} / 2 ; \omega+\theta_{t} / 2\right]$. Besides, recall that the measure of $\Omega_{t}$ is $N_{t}$ (the "number of sectors"); since $\theta_{t}$ is the measure of the subset of sectors of $\Omega_{t}$ that receive knowledge $\chi_{\omega t}$, it is reasonable to assume that $\theta_{t}$ is function of $N_{t}$ which is bounded above by $N_{t}$. Finally, since $N_{t}=\gamma L_{t}$, it is equivalent to assume that $\theta_{t}=\theta\left(L_{t}\right)$. Some additional assumptions on this function, in particular on the signs of $\theta^{\prime}\left(L_{t}\right)$ and $\theta^{\prime \prime}\left(L_{t}\right)$, will be made in Section 4 below. In particular, we will successively consider $\theta\left(L_{t}\right)=\theta$, $\theta^{\prime}\left(L_{t}\right)>0$ (and $\theta^{\prime \prime}\left(L_{t}\right)<0$ ), or $\theta^{\prime}\left(L_{t}\right)<0$, and we will study the consequence of these different assumptions on scale effects. For instance, we will show that $\theta^{\prime}\left(L_{t}\right)>0$ implies scale effects and that their significance decreases over time if $\theta^{\prime \prime}\left(L_{t}\right)<0$.

Let us denote by $\Theta_{\omega t}$ the "scope of diffusion" of the stock of knowledge $\chi_{\omega t}$, which is defined as the measure of the subset of sectors of $\Omega_{t}$ on which knowledge $\chi_{\omega t}$ diffuses. $\Theta_{\omega t}$ can take the value $N_{t}=\gamma L_{t}$ with probability $p_{G}$, the value 0 with probability $p_{s}$, and the value $\theta\left(L_{t}\right)$ with probability $p_{m}$. Consequently, at each date $t$, the average scope of knowledge diffusion from any sector $\omega$ is

$$
\mathbb{E}\left[\Theta_{\omega t}\right] \equiv \mathbb{E}\left[\Theta_{t}\right]=p_{G} \gamma L_{t}+p_{m} \theta\left(L_{t}\right), \forall \omega \in \Omega_{t}
$$

$\mathbb{E}\left[\Theta_{t}\right]$ can be interpreted as the intensity of inter-sectoral knowledge spillovers. It is comprised between the intensities of knowledge spillovers characterizing the two polar cases introduced in 
Section 2. Firstly, if $p_{G}=1$ (i.e. $p_{s}=p_{m}=0$ ), then $\mathbb{E}\left[\Theta_{t}\right]=N_{t}=\gamma L_{t}$ : in this case - in fact, the polar case $i$ ) described at the end of Subsection 2.1 - there would be only GPTs (in other words, there would be only global inter-sectoral spillovers). Secondly, if $p_{s}=1$ (i.e. $p_{G}=p_{m}=0$ ), then $\mathbb{E}\left[\Theta_{t}\right]=0$ : in this case - in fact, the polar case $\left.i i\right)$ - there would be no inter-sectoral knowledge diffusion (this would be an economy in which spillovers would be only intra-sectoral).

\subsubsection{Receiving knowledge: how are the pools of knowledge shaped?}

Now, we aim to provide a formal expression of the pool of knowledge used in each sector $\omega$. In that respect, we investigate how these pools are shaped given the formalization of knowledge diffusion introduced in 3.1.1. Specifically, one has to determine the impact of each of the three types of knowledge diffusion on any given sector. Let us consider the standard symmetric case in which $\chi_{\omega t}=\chi_{t}, \forall \omega \in \Omega_{t}$. The R\&D activity of any sector $\omega, \omega \in \Omega_{t}$, uses knowledge produced in three disjoint subsets of the Salop circle. First, sector $\omega$ always uses the stock of knowledge $\chi_{\omega t}=\chi_{t}$ produced by its own R\&D activity. Second, sector $\omega$ can also use the stock of knowledge produced by sectors that are located in the neighborhood $\left[\omega-\theta\left(L_{t}\right) / 2 ; \omega+\theta\left(L_{t}\right) / 2\right]$ on the Salop circle. On this subset of measure $\theta\left(L_{t}\right)$, the knowledge that could potentially be used by sector $\omega$ is equal to $\left[\theta\left(L_{t}\right) \chi_{t}-\chi_{t}\right]$ (the stock $\chi_{\omega t}=\chi_{t}$ is subtracted since it has already been taken into account). The knowledge effectively used here is either diffused from sectors producing medium knowledge which are close enough to sector $\omega$ (i.e. located in the neighborhood), or from any sector producing GPTs also located in this neighborhood. Since the proportions of these sectors are respectively $p_{m}$ and $p_{G}$, the total stock of knowledge diffused from this neighborhood and effectively used by sector $\omega$ is thus $\left(p_{m}+p_{G}\right)\left[\theta\left(L_{t}\right) \chi_{t}-\chi_{t}\right]$. Third, sector $\omega$ can finally use the stock of knowledge produced by sectors located outside the neighborhood $\left[\omega-\theta\left(L_{t}\right) / 2 ; \omega+\theta\left(L_{t}\right) / 2\right]$, that is by sectors located on the subset of the Salop circle of measure $N_{t}-\theta\left(L_{t}\right)$. The knowledge diffused from this subset that could be potentially used by sector $\omega$ is equal to $\left[N_{t}-\theta\left(L_{t}\right)\right] \chi_{t}$. Since within this subset only the knowledge diffused by sectors producing GPTs reaches sector $\omega$, and since the proportion of such sectors is $p_{G}$, the total stock of knowledge diffused from this subset and effectively used by sector $\omega$ is thus $p_{G}\left[N_{t}-\theta\left(L_{t}\right)\right] \chi_{t}$. Finally, the pool of knowledge used by any sector $\omega$ is $\mathcal{P}_{\omega t}=\mathcal{P}_{t}=\chi_{t}+\left(p_{m}+p_{G}\right)\left[\theta\left(L_{t}\right) \chi_{t}-\chi_{t}\right]+p_{G}\left[N_{t}-\theta\left(L_{t}\right)\right] \chi_{t}, \forall \omega \in \Omega_{t}$. After simplification, one gets $\mathcal{P}_{\omega t}=\mathcal{P}_{t}=p_{s} \chi_{t}+p_{m} \theta\left(L_{t}\right) \chi_{t}+p_{G} \mathcal{K}_{t}, \forall \omega \in \Omega_{t}$, where $\mathcal{K}_{t}=N_{t} \chi_{t}=\gamma L_{t} \chi_{t}$ is the whole disposable stock knowledge in the economy introduced in (1). The following proposition summarizes the main results related to knowledge diffusion and to the pools of knowledge that stem from it.

Proposition 3. At each date $t$, for any sector $\omega$, the average scope of knowledge diffusion from this sector is

$$
\mathbb{E}\left[\Theta_{\omega t}\right] \equiv \mathbb{E}\left[\Theta_{t}\right]=p_{G} \gamma L_{t}+p_{m} \theta\left(L_{t}\right), \forall \omega \in \Omega_{t},
$$

and the pool of knowledge used by this sector is

$$
\begin{aligned}
\mathcal{P}_{\omega t}=\mathcal{P}_{t}=p_{s} \chi_{t}+p_{m} \theta\left(L_{t}\right) \chi_{t}+p_{G} \mathcal{K}_{t}, \forall \omega \in \Omega_{t}, \\
\text { where } \mathcal{K}_{t}=N_{t} \chi_{t}=\gamma L_{t} \chi_{t}, \text { that is } \mathcal{P}_{t}=\left(p_{s}+\mathbb{E}\left[\Theta_{t}\right]\right) \chi_{t}
\end{aligned}
$$

Note that the formalization that we have presented so far can be related to the concept of "technological distance" introduced in particular in Peretto \& Smulders (2002). Indeed, they consider that the "extent to which a firm can take advantage of the public knowledge created by other firms decreases with the technological distance between the creator and the user of such knowledge". We will develop the link between scale effects, knowledge diffusion and technological distance further in 4.2.4.

Before completing the presentation of the model, let us return to the two polar cases studied previously, in particular in Proposition 2. According to Proposition 3, on has the following results.

i) In an economy in which there would only be GPTs (i.e. if $p_{G}=1$ ), the expected scope of knowledge diffusion (i.e. the intensity of knowledge spillovers) would be maximum: from (6) one has $\mathbb{E}\left[\Theta_{t}\right]=N_{t}=\gamma L_{t}$. The pool of knowledge used in each sector would comprise the whole disposable knowledge in the economy: from (7), one has $\mathcal{P}_{\omega t}=\mathcal{K}_{t}, \forall \omega \in \Omega_{t}$. Accordingly, from Proposition 1, knowledge would accumulate along with $\dot{\chi}_{\omega t}=\lambda \sigma l_{\omega t} \mathcal{K}_{t}$, $\forall \omega \in \Omega_{t}$. 
ii) In economy in which there would be only "sector specific knowledge" (i.e. if $p_{s}=1$ ) - that is, in which spillovers would be only intra-sectoral - the expected scope of knowledge diffusion would be minimum: from $(6), \mathbb{E}\left[\Theta_{t}\right]=0$. Because there are no spillovers between the sectors' R\&D activities here, the pool of knowledge used in each sector would comprise only the knowledge accumulated in the sector so far: from (7), $\mathcal{P}_{\omega t}=\chi_{\omega t}, \forall \omega \in \Omega_{t}$. Then, from Proposition 1, knowledge would accumulate along with $\dot{\chi}_{\omega t}=\lambda \sigma l_{\omega t} \chi_{\omega t}, \forall \omega \in \Omega_{t}$.

In Section 4, when studying the link between knowledge diffusion and scale effects, we will consider successively different assumptions on the probabilities $p_{G}, p_{s}$ and $p_{m}$, and on the derivatives $\theta^{\prime}\left(L_{t}\right)$ and $\theta^{\prime \prime}\left(L_{t}\right)$. Depending on the assumptions considered, we will in particular get a scale-invariant model, or not.

\subsection{Other assumptions: standard Schumpeterian growth framework}

In the previous section, we presented the way knowledge accumulates, the key part played by the pools of knowledge in this process, and we formalized how these pools arise from knowledge diffusion. Now, we present the remainder of the assumptions which are rather standard in Schumpeterian growth theory.

\section{Preferences and technologies}

Each household is modelled as a dynastic family which maximizes the discounted utility ${ }^{12} \mathcal{U}=$ $\int_{0}^{\infty} L_{t} u\left(c_{t}\right) e^{-\rho t} d t$, where $\rho>n$ is the common subjective discount rate and $u\left(c_{t}\right)$ is the individual instantaneous utility at time $t$, which is given by $u\left(c_{t}\right)=\ln \left(c_{t}\right) .{ }^{13}$ Given Assumption 1 , the population of workers in the economy at time $t$ is $L_{t}=e^{n t}$. Intertemporal preferences are thus given by

$$
\mathcal{U}=\int_{0}^{\infty} \ln \left(c_{t}\right) e^{(n-\rho) t} d t
$$

At each date $t$, each of the $L_{t}$ identical households is endowed with one unit of labor that is supplied inelastically. The total quantity of labor $L_{t}$ is used to produce the final good and in R\&D activities. Hence, the labor constraint is

$$
L_{t}=L_{t}^{Y}+\int_{\Omega_{t}} l_{\omega t} d \omega
$$

Besides labor, the production of the final good requires the use of all available intermediate goods, each of which is associated with its own level of knowledge. The final good production technology is

$$
Y_{t}=\left(L_{t}^{Y}\right)^{1-\alpha} \int_{\Omega_{t}} \chi_{\omega t}\left(x_{\omega t}\right)^{\alpha} d \omega, 0<\alpha<1,
$$

The final good has two competing uses. Firstly, it is used in the production of intermediate goods along with

$$
x_{\omega t}=\frac{y_{\omega t}}{\chi_{\omega t}}, \omega \in \Omega_{t},
$$

where $y_{\omega t}$ is the quantity of final good used to produce $x_{\omega t}$ units of intermediate good $\omega$. This technology illustrates the increasing complexity in the production of intermediate goods: as the quality of a given intermediate good increases, its production requires more resources. Secondly, it is consumed by the representative household in quantity $c_{t}$. One gets the following constraint on the final good market:

$$
Y_{t}=L_{t} c_{t}+\int_{\Omega_{t}} y_{\omega t} d \omega
$$

\footnotetext{
${ }^{12}$ Barro \& Sala-i-Martin (1995, Ch. 2) provide more details on this formulation of the households behavior within the context of the Ramsey model of growth. See also Segerstrom (1998).

${ }^{13}$ The results are robust if one considers a C.E.S. instantaneous utility function of parameter $\varepsilon, u\left(c_{t}\right)=\frac{c_{t}^{1-\varepsilon}}{1-\varepsilon}$.
} 


\section{Decentralized economy}

We consider a decentralized economy with creative destruction which is in direct line with the equilibrium introduced by Aghion \& Howitt (1992). ${ }^{14}$

The main features of this standard Schumpeterian framework are the following. Once an innovation occurs, the innovator is granted an infinitely-lived patent; she monopolizes the production and sale of the intermediate good (which embodies knowledge) until replaced by the next innovator. This equilibrium involves two market failures. The first one, which results from the presence of monopolies, can be corrected by an ad valorem subsidy $\psi$ on each intermediate good demand. The second one relates to the externality triggered by the fact that there is no market for knowledge; it can be corrected by a public tool $\varphi$ which can consist in a subsidy or in a tax on the profits of R\&D activities. Normalizing the price of final good to one, and denoting the interest rate, the wage, and the price of intermediate good $\omega$ at date $t$ by $r_{t}, w_{t}$, and $q_{\omega t}\left(\omega \in \Omega_{t}\right)$, respectively, the set of Schumpeterian equilibria as defined as follows.

Definition 1. At each vector of public policy tools $(\psi, \varphi)$ is associated a particular Schumpeterian equilibrium. It consists of time paths of set of quantities

$$
\left\{\left(c_{t}(\psi, \varphi), Y_{t}(\psi, \varphi),\left\{l_{\omega t}(\psi, \varphi)\right\}_{\omega \in \Omega}, L_{t}^{Y}(\psi, \varphi),\left\{x_{\omega t}(\psi, \varphi)\right\}_{\omega \in \Omega},\left\{\chi_{\omega t}(\psi, \varphi)\right\}_{\omega \in \Omega}\right)\right\}_{t=0}^{\infty}
$$

and of prices

$$
\left\{\left(r_{t}(\psi, \varphi), w_{t}(\psi, \varphi),\left\{q_{\omega t}(\psi, \varphi)\right\}_{\omega \in \Omega}\right)\right\}_{t=0}^{\infty}
$$

such that: the representative household maximizes her utility; firms maximize their profits; the final good market, the financial market and the labor market are perfectly competitive and clear; on each intermediate good market, the innovator is granted an infinitely-lived patent and monopolizes the production and sale until replaced by the next innovator; and there is free entry on each RGD activity (i.e. the zero profit condition holds for each R\&D activity).

In the following proposition, we present exhaustively the variables at equilibrium. In Section 4 (Proposition 5), we will only retain the ones that are necessary to tackle the issue of scale effects.

Proposition 4. At each date $t$, the set of Schumpeterian equilibria à la Aghion E Howitt is characterized as follows.

Quantities (levels and growth rates).

The labor partition is

$$
\begin{gathered}
l_{\omega t}(\psi, \varphi)=l(\psi, \varphi)=\frac{1}{\gamma}-\frac{\lambda / \gamma+\rho}{\lambda\left(1+\frac{1+\varphi}{1-\psi} \alpha\right)}, \forall \omega \in \Omega_{t}, \\
\text { and } L_{t}^{Y}(\psi, \varphi)=L_{t}-N_{t} l(\psi, \varphi)=[1-\gamma l(\psi, \varphi)] L_{t}
\end{gathered}
$$

The quantity of intermediate $\omega$ is

$$
x_{\omega t}(\psi, \varphi)=x_{t}(\psi, \varphi)=\left(\frac{\alpha^{2}}{1-\psi}\right)^{\frac{1}{1-\alpha}} L_{t}^{Y}(\psi, \varphi), \forall \omega \in \Omega_{t}
$$

The growth rate of knowledge in each sector $\omega$ is

$$
g_{\chi_{\omega t}}(\psi, \varphi)=g_{\chi_{t}}(\psi, \varphi)=\lambda \sigma\left(p_{s}+\mathbb{E}\left[\Theta_{t}\right]\right) l(\psi, \varphi), \forall \omega \in \Omega_{t}
$$

The growth rate of the economy is

$$
g_{c_{t}}(\psi, \varphi)=g_{Y_{t}}(\psi, \varphi)-n=g_{\mathcal{K}_{t}}(\psi, \varphi) \equiv g_{t}(\psi, \varphi)=g_{\chi_{t}}(\psi, \varphi)+n
$$

Prices. The equilibrium interest rate, wage and prices of intermediate goods (price of final good normalized to one) are

$$
\begin{gathered}
r_{t}(\psi, \varphi)=g_{t}(\psi, \varphi)+\rho, w_{t}(\psi, \varphi)=(1-\alpha)\left(\frac{\alpha^{2}}{1-\psi}\right)^{\frac{\alpha}{1-\alpha}} \mathcal{K}_{t}(\psi, \varphi) \text { and } \\
q_{\omega t}(\psi, \varphi)=q_{t}(\psi, \varphi)=\frac{\mathcal{K}_{t}(\psi, \varphi)}{\alpha \gamma L_{t}}, \forall \omega \in \Omega_{t}, \text { where } \mathcal{K}_{t}(\psi, \varphi)=e^{\int_{0}^{t} g_{s}(\psi, \varphi) d s}
\end{gathered}
$$

\footnotetext{
${ }^{14} \mathrm{We}$ implicitly consider the case of drastic innovations. One could characterize the condition on the parameters, in particular the ones related to knowledge diffusion, under which innovations are drastic or non drastic. This analysis can be found in a more elementary version of this model: see Gray \& Grimaud (2016).
} 
Proof. See Appendix 6.2.

This proposition shows that the variables of the economy depend on the vector of public policy tools $(\psi, \varphi)$. In particular, these public policies have an impact on the growth rate. In this respect, this model is in line with the ones developed in the fully endogenous growth theory (e.g. Aghion \& Howitt 1998; Dinopoulos \& Thompson 1998; Peretto 1998; Young 1998; Howitt 1999; Peretto \& Smulders 2002; Ha \& Howitt 2007). This literature has indeed provided endogenous growth models in which public policies affect the economy; besides, they did so while suppressing the non desirable property of scale effects. In Section 4 below, we will explain that scale effects were canceled by assuming (explicitly or implicitly) no inter-sectoral knowledge diffusion.

Clearly, our fully endogenous growth model might exhibit scale effects. Indeed, the growth rate of the economy $g_{t}(\psi, \varphi)$ can potentially depend on the size of the economy $L_{t}$. This point is examined in detail in Section 4 below, in which we revisit the issue of scale effects by revealing its link with knowledge diffusion.

\section{Scale effects property revisited}

The following proposition extracts from Proposition 4 the basic results that enable us to analyze along the whole present section the issue of scale effect under the light of knowledge diffusion.

Proposition 5. If $p_{G} \geq 0, p_{m} \geq 0$, and $\theta_{t}=\theta\left(L_{t}\right)$, where $\theta($.$) is a function of class C^{2}$, then the main results to study the scale effects property are the following.

In each sector $\omega$,

- the intensity of inter-sectoral knowledge diffusion, the pool of knowledge used by R\&D activity, and the quantity of labor devoted to $R \& D$ are respectively

$$
\begin{gathered}
\mathbb{E}\left[\Theta_{\omega t}\right]=\mathbb{E}\left[\Theta_{t}\right]=p_{G} \gamma L_{t}+p_{m} \theta\left(L_{t}\right), \mathcal{P}_{\omega t}(\psi, \varphi)=\mathcal{P}_{t}(\psi, \varphi)=\left(p_{s}+\mathbb{E}\left[\Theta_{t}\right]\right) \chi_{t}(\psi, \varphi), \\
\text { and } l_{\omega t}(\psi, \varphi)=l(\psi, \varphi)=\frac{1}{\gamma}-\frac{\lambda / \gamma+\rho}{\lambda\left(1+\frac{1+\varphi}{1-\psi} \alpha\right)} ;
\end{gathered}
$$

- the law of knowledge accumulation, and the marginal productivity of labor in R\&D activity are respectively

$$
\begin{gathered}
\dot{\chi}_{\omega t}(\psi, \varphi)=\dot{\chi}_{t}(\psi, \varphi)=\lambda \sigma l_{\omega t}(\psi, \varphi) \mathcal{P}_{\omega t}(\psi, \varphi), \\
\text { and } \frac{\partial \dot{\chi}_{\omega t}}{\partial l_{\omega t}}(\psi, \varphi)=\lambda \sigma \mathcal{P}_{\omega t}(\psi, \varphi), \forall \omega \in \Omega_{t} .
\end{gathered}
$$

The growth rate of the economy is

$$
g_{t}(\psi, \varphi)=\lambda \sigma l(\psi, \varphi)\left(p_{s}+\mathbb{E}\left[\Theta_{t}\right]\right)+n
$$

As sated above, the present model might exhibit scale effect. ${ }^{15}$ What is their origin? As seen in Proposition 5, the R\&D effort (the number of researchers) in each sector, $l(\psi, \varphi)$, is here independent of $L_{t}$. In other words, scale effects do not stem from the fact that an increase in the size of the economy implies a rise in the number of researchers, leading to a higher growth rate. Thus scale effects can result only from the presence of $\mathbb{E}\left[\Theta_{t}\right]$, the intensity of inter-sectoral knowledge diffusion, in $g_{t}(\psi, \varphi)$. Finally, $\mathbb{E}\left[\Theta_{t}\right]$ potentially depends positively on the size of the economy $L_{t}$ through two fundamental factors (each of which corresponding to a specific type of knowledge diffusion). First, the term related to the presence of GPTs, $p_{G} \gamma L_{t}$. Second, the term related to the presence of medium knowledge, $p_{m} \theta\left(L_{t}\right)$.

It appears here that there seems to be a fundamental contradiction between the absence of scale effects and the presence of inter-sectoral knowledge diffusion. Indeed, on the one hand - as argued for instance by Jones (1995a) - the presence of scale effects is clearly counterfactual. On

\footnotetext{
${ }^{15}$ More precisely, the model might exhibit strong scale effects $\left(g_{t}(\psi, \varphi)\right.$ may depend on $\left.L_{t}\right)$, and it exhibits weak scale effects $\left(g_{t}(\psi, \varphi)\right.$ depends on $\left.n\right)$ as long as $n>0$. Note that, contrary to semi-endogenous growth models, the growth rate is still positive even if $n=0$. As mentioned above in the introduction (see footnote 4 ), this paper focuses on the issue of strong scale effects; accordingly we refer to them as to "scale effects".
} 
the other hand, the existence of inter-sectoral knowledge diffusion is indisputable: it has effectively been highlighted by many empirical studies (e.g. Griliches 1992; Griliches 1995; Hall, Mairesse \& Mohnen 2010) and it has also been emphasized in the endogenous growth theory (as stated, for instance, in Aghion \& Howitt 1998, Ch. 3, it is generally agreed that new pieces of knowledge "diffuse gradually, through a process in which one sector gets ideas from the research and experience of others"). ${ }^{16}$

Our aim now is to investigate whether this contradiction can be overcome. More precisely, we want to determine whether it is possible to develop a fully endogenous Schumpeterian growth model that maintains inter-sectoral knowledge diffusion while remaining in accordance with the empirical facts regarding scale effects. In order to tackle this issue and to go further in the analysis of the link between scale effects and knowledge diffusion, we introduce the following definition in which we provide a measure of the impact of the size of the economy (as measured by the population level $L_{t}$ ) on the growth rate:

Definition 2. The measure of scale effects is

$$
\mathcal{S}_{t}=\frac{\partial g_{t}(\psi, \varphi)}{\partial L_{t}}=\lambda \sigma l(\psi, \varphi) \frac{\partial \mathbb{E}\left[\Theta_{t}\right]}{\partial L_{t}}=\lambda \sigma l(\psi, \varphi)\left[p_{G} \gamma+p_{m} \theta^{\prime}\left(L_{t}\right)\right]
$$

As mentioned above, two types of inter-sectoral knowledge diffusion have an effect on the significance of scale effects:

1. Scale effects can result from the presence of GPTs (see $\left.p_{G} \gamma\right)$.

2. Scale effects can also be a consequence of the presence of medium knowledge (i.e. knowledge impacting a more or less wide range of sectors): $\mathcal{S}_{t}$ depends both on the probability $p_{m}$ of arrival of this type of knowledge, and on the derivative $\theta^{\prime}\left(L_{t}\right)$, which measures to what extent the diffusion of this knowledge is affected by the size of the economy, $N_{t}=\gamma L_{t}$. Regarding the sign of this derivative, we argue that it depends on two opposite effects.

(a) On the one hand, one could argue that, as the number of sectors $N_{t}$ increases, the knowledge produced in any given sector can potentially influence more sectors. This "expanding effect" tends to increase $\theta\left(L_{t}\right)$, that is the measure of the subset of sectors that use medium knowledge $\left[\omega-\theta\left(L_{t}\right) / 2 ; \omega+\theta\left(L_{t}\right) / 2\right]$.

(b) On the other hand, it could also be argued that, as its size increases, the economy becomes more complex, which implies that sectors specialize and become less likely to interact. This "specialization effect" tends to reduce $\theta\left(L_{t}\right)$.

If the expanding effect overcomes the specialization effect, then the derivative $\theta^{\prime}\left(L_{t}\right)$ is positive. Conversely, if the specialization effect overcomes the expanding effect, then $\theta^{\prime}\left(L_{t}\right)$ is negative.

In this section, we investigate under which reasonable assumptions on knowledge diffusion one can obtain a Schumpeterian growth model in adequation with well established empirical evidences, namely a model with some knowledge diffusion that does not exhibit significant scale effects and in which public policies have an impact on the growth rate. In Subsection 4.1, we return more rigourously to the intuition given in Section 2 along which if there is no inter-sectoral knowledge diffusion, there are no scale effects. In Subsection 4.2, we introduce progressively inter-sectoral knowledge diffusion and we study its impact on scale effects; this will allows us to see how the contradiction aforementioned can be overcome.

\subsection{No inter-sectoral knowledge diffusion $\left(\mathbb{E}\left[\Theta_{t}\right]=0\right)$ : no scale effects $\left(\mathcal{S}_{t}=0\right)$}

In the intuitive analysis provided in Section 2, we stated the following: if there is no inter-sectoral knowledge diffusion (i.e. if sectors do not share knowledge), there are no scale effects. This result is now proven rigourously within a standard Schumpeterian model in which we have formalized

\footnotetext{
${ }^{16}$ Similar statements can be found, for instance, in Scotchmer (1991), Acemoglu (2009, Ch. 12), or in Chantrel, Grimaud \& Tournemaine (2010).
} 
explicitly knowledge diffusion. In this model, the absence of inter-sectoral knowledge diffusion corresponds to the case in which the sectors produce neither GPTs (i.e. $p_{G}=0$ ) nor medium knowledge (i.e. $p_{m}=0$ ), that is to the case in which all sectors produce only sector specific knowledge $\left(\right.$ i.e. $\left.p_{s}=1\right)$ :

Proposition 6. If $p_{G}=p_{m}=0$ (i.e. $p_{s}=1$ ), or equivalently if the intensity of inter-sectoral knowledge diffusion is $\mathbb{E}\left[\Theta_{\omega t}\right] \equiv \mathbb{E}\left[\Theta_{t}\right]=0, \forall \omega \in \Omega_{t}$, there is only intra-sectoral knowledge diffusion, the growth rate is $g_{t}(\psi, \varphi)=\lambda \sigma l(\psi, \varphi)+n$, and the measure of scale effects $\mathcal{S}_{t}$ is nil. There are no scale effects because there is no inter-sectoral knowledge diffusion.

Proof. The results of Proposition 6 are obtained by assuming $p_{G}=p_{m}=0$ (and thus $p_{s}=1$ ) in Proposition 5 and in (14).

This proposition shows that a sufficient condition for fully endogenous growth models not to exhibit scale effects is the absence of inter-sectoral knowledge diffusion. We previously provided some details on this polar case: in Proposition 2 - ii), we assumed directly that the pool of knowledge used in each sector comprised only the knowledge accumulated so far in this sector. The following corollary shows that this assumption - which is directly made on the pools - is equivalent to assuming no inter-sectoral knowledge diffusion.

Corollary 1. The assumption of no inter-sectoral knowledge diffusion is equivalent to assuming that each sector uses a pool of knowledge constituted solely of the stock of knowledge produced within this sector so far:

$$
\mathbb{E}\left[\Theta_{t}\right]=0 \Leftrightarrow \mathcal{P}_{\omega t}=\chi_{\omega t}=\chi_{t}, \forall \omega \in \Omega_{t}
$$

Proof. In the framework we propose, under the symmetry assumption, the most general expression (i.e. considering any possible type of knowledge diffusion) of the pool of knowledge used in each sector (given in Proposition 3) is $\mathcal{P}_{\omega t}=\left(p_{s}+\mathbb{E}\left[\Theta_{t}\right]\right) \chi_{t}, \forall \omega \in \Omega_{t}$. Assuming that $\mathcal{P}_{\omega t}=\chi_{t}, \forall \omega \in \Omega_{t}$, is thus equivalent to assuming that $p_{s}+\mathbb{E}\left[\Theta_{t}\right]=1, \forall t \Leftrightarrow p_{s}+p_{G} \gamma L_{t}+p_{m} \theta\left(L_{t}\right)=$ $1, \forall t \Leftrightarrow p_{G}\left(N_{t}-1\right)+p_{m}\left[\theta\left(L_{t}\right)-1\right]=0, \forall t$. Since $1<\theta\left(L_{t}\right)<N_{t}$, this equality holds if and only if $p_{s}=p_{G}=0 \Leftrightarrow \mathbb{E}\left[\Theta_{t}\right]=0, \forall t$.

As explained above in Proposition 2 and in its comments, the basic arguments for understanding the result of Proposition 6 are the following. As seen in its corollary, because there is no inter-sectoral diffusion of knowledge, the knowledge produced in any given sector is not used by any other sector. Therefore, in each sector, the pool used by R\&D and the law of knowledge accumulation are $\mathcal{P}_{\omega t}=\chi_{t}$ and $\dot{\chi}_{\omega t}=\dot{\chi}_{t}=\lambda \sigma l_{t} \chi_{t}, \forall \omega \in \Omega_{t}$, respectively. Accordingly, the marginal productivity of labor in R\&D is $\partial \dot{\chi}_{\omega t} / \partial l_{\omega t}=\lambda \sigma \chi_{t}$. It clearly appears that, because there is no inter-sectoral knowledge diffusion, the marginal productivity of labor does not depend on the size of the economy, $L_{t}$. That is why there are no scale effects.

The result of Proposition 6 echoes to the way scale-invariancy is achieved in the fully endogenous growth theory. Indeed, it will be explained below that, in the related literature, scale effects have been removed by wiping out inter-sectoral knowledge diffusion.

Surveys on the scale effects issue by Jones (1999), Laincz \& Peretto (2006), Dinopoulos \& Sener (2007), or Ha \& Howitt (2007), explain that a "variety expansion mechanism" has been introduced in order to remove scale effects from endogenous growth models while maintaining the effects of public policies. The basic underlying idea in this range of endogenous growth models follows from Young (1998)'s insight that, as population grows, the proliferation of sectors reduces the efficiency of R\&D activities in improving the quality of an existing product because the R\&D effort is diluted in more sectors. The formalization typically considered in this respect relies on two assumptions. The first one is the proportionality between the population level and the number of sectors that was presented and discussed above in Assumption 1. The second one relates to the formalization of the process of knowledge accumulation (more precisely to the intensity of knowledge spillovers), two types of which can a priori be distinguished (in fact, we show below that they are eventually similar since they both consider no inter-sectoral knowledge diffusion). 
1) Intra-sectoral knowledge spillovers only. In Segerstrom (1998), Peretto (1999), Acemoglu (2009, Ch. 14), or Aghion \& Howitt (2009, Ch. 4), among others, it is implicitly assumed that the pool of knowledge used in each sector comprises only the knowledge previously accumulated within this sector. Using our notations, the considered knowledge production function in each sector is: $\dot{\chi}_{\omega t}=\lambda \sigma l_{\omega t} \mathcal{P}_{\omega t}$, where $\mathcal{P}_{\omega t}=\chi_{\omega t}, \forall \omega \in \Omega_{t}$; there is no inter-sectoral knowledge diffusion in these models, as in the case presented in the Corollary 1 of Proposition 6.

2) Knowledge spillovers depending on average knowledge. A second type of scale-invariant fully endogenous growth models can be identified. The models of Dinopoulos \& Thompson (1998), Peretto (1998), Howitt (1999), or Li (2000 and 2003), among others, consider firm-specific knowledge production functions such that, as stated by Laincz \& Peretto (2006), "spillovers depend on average knowledge". In other words, as argued in the hereinbefore overviews, many scale-invariant fully endogenous growth models assume that, in each sector, new knowledge is produced using the average knowledge across all sectors. ${ }^{17}$ For instance, Laincz \& Peretto (2006) formalize this assumption in equation 9 of their paper. One can equivalently refer to equations 7 and 9 in Jones (1999); to equations 13 and 14 in Dinopoulos \& Sener (2007); to equation 5 in Ha \& Howitt (2007); or to the framework used in Aghion \& Howitt (2009, Ch. 4). Using our notations, this assumption amounts to considering the following knowledge production function in each sector:

$$
\dot{\chi}_{\omega t}=\lambda \sigma l_{\omega t} \mathcal{P}_{\omega t}, \text { where } \mathcal{P}_{\omega t}=\int_{\Omega_{t}} \frac{\chi_{h t}}{N_{t}} d h, \forall \omega \in \Omega_{t}
$$

Hence, this formalization leans on a "normalization" of the pool of disposable knowledge by the number of sectors, and hence equivalently by the population level (because of the proportionality assumption). As shown in the following corollary, assuming that new knowledge is produced using the average knowledge across all sectors boils down to considering that the pool of knowledge in which each sector draws from to produce new knowledge is reduced to the knowledge which is specific to this sector.

Corollary 2. In the usual symmetric case, the assumption along which the pool of knowledge used in each sector consists in the average knowledge in the economy is equivalent to assuming that each sector uses a pool of knowledge constituted solely of the stock of knowledge produced within this sector so far:

$$
\mathcal{P}_{\omega t}=\int_{\Omega_{t}} \frac{\chi_{h t}}{N_{t}} d h=\chi_{t}, \forall \omega \in \Omega_{t}
$$

Proof. From (15) and (1), one has $\mathcal{P}_{\omega t}=\frac{1}{N_{t}} \int_{0}^{N_{t}} \chi_{h t} d h=\mathcal{K}_{t} / N_{t}$. Moreover, under the standard assumption of symmetry in which $\chi_{\omega t}=\chi_{t}, \forall \omega \in \Omega_{t}$, (1) writes $\mathcal{K}_{t}=N_{t} \chi_{t}$. Consequently, $\mathcal{P}_{\omega t}=\chi_{t}, \forall \omega \in \Omega_{t}$.

Corollaries 1 and 2 show that, considering knowledge spillovers depending on average knowledge is equivalent to consider implicitly that there is no inter-sectoral knowledge diffusion, that is to assume $\mathbb{E}\left[\Theta_{t}\right]=0$. From Proposition 2, Corollary 1 and their comments we know that, in this case, there are no scale effects because the marginal productivity of labor in R\&D does not depend on the size of the economy.

In this subsection, we have shown that a sufficient condition to suppress scale effects is to assume that there is no inter-sectoral knowledge diffusion. Moreover, we have seen that this is the assumption which is made explicitly or implicitly in most of the scale-invariant fully endogenous growth theory so far. ${ }^{18}$ However, one can think that wiping out inter-sectoral knowledge diffusion

\footnotetext{
${ }^{17}$ The argument commonly put forward to justify the normalization by the size of the economy follows from the assumptions that the number of sectors and the population level are proportional (and both measure the size of the economy), and that the number of sectors is a measure of R\&D difficulty. Then, assuming that each sector uses only the average knowledge is a way to account for the fact that, as R\&D difficulty increases, the same level of R\&D investment generates a lower production of knowledge.

${ }^{18}$ A similar statement is provided in Peretto \& Smulders (2002): "R\&D productivity depends on some measure of accumulated public knowledge that is independent of the number of firms and hence of the scale of the economy.
} 
from models originally considering some type of interaction between sectors amounts to undermining the fundamental property of non rivalry of knowledge. It is clear that this is in contradiction with the common view on how knowledge springs into existence. Indeed, as mentioned above, the significance of the interactions between sectors resulting from this non rivalry has been emphasized both theoretically and empirically.

Finally, the question raised here is the following: is it possible to suppress the non desirable property of scale effects while maintaining the effects of public policies, but without removing knowledge diffusion? In order to study this apparent paradox, we will consider in Subsection 4.2 that there is some inter-sectoral knowledge diffusion. Formally, it will be assumed that $\mathbb{E}\left[\Theta_{t}\right] \neq 0$ from now on.

\subsection{Are the presence of inter-sectoral knowledge diffusion $\left(\mathbb{E}\left[\Theta_{t}\right] \neq 0\right)$ and the absence of scale effects $\left(\mathcal{S}_{t}=0\right)$ compatible?}

Our goal now is to study further the link between knowledge diffusion and scale effects and, in particular, to determine whether the paradox mentioned above can be overcome. First, in 4.2.1, we show that it is possible to build a fully endogenous growth model in which scale effects are cancelled while maintaining some inter-sectoral knowledge diffusion (the type of diffusion considered here is quite simple in that it does not allow for the possible occurrence of GPTs and in that medium knowledge diffusion does not vary with the size of the economy - in the remaining of the section, we then consider more general assumptions). Second, in 4.2.2, we isolate the impact of GPTs on scale effects. Third, in 4.2.3, we isolate the impact of the diffusion of medium knowledge on scale effects by introducing more general assumptions on how this diffusion expands (or contracts) when the size of the economy increases. Finally, in 4.2.4, we summarize under which reasonable assumptions on inter-sectoral knowledge diffusion (specifically on GPTs and on medium knowledge diffusion) one can obtain a Schumpeterian growth model in adequation with commonly agreed empirical facts, that is a model that does not exhibit scale effects (or at least not significant ones), in which public policies have an impact on the growth rate, in which GPTs can occur, and in which medium knowledge diffusion may be affected by the fact that the size of the economy increases.

\subsubsection{A scale-invariant fully endogenous growth model with inter-sectoral knowledge diffusion}

Using our formalization, it is in fact quite easy to exhibit a fully endogenous growth model in which there is some inter-sectoral knowledge diffusion, but no scale effects. One just has to consider a case in which $\mathbb{E}\left[\Theta_{t}\right]$ is independent of $L_{t}$. For that purpose, it is sufficient to make two assumptions. First, there are no GPTs: $p_{G}=0$. Second, there are some sectors producing medium knowledge, the diffusion of which being constant: $p_{m}>0$ and $\theta\left(L_{t}\right)=\theta$, where $\theta>1$ is a constant parameter (i.e. the expanding and specialization effects exactly compensate each others). This leads to the following proposition:

Proposition 7. If $p_{G}=0, p_{m}>0$ and $\theta\left(L_{t}\right)=\theta, \theta>1$, then the intensity of inter-sectoral knowledge diffusion is $\mathbb{E}\left[\Theta_{\omega t}\right] \equiv \mathbb{E}\left[\Theta_{t}\right]=p_{m} \theta>0, \forall \omega \in \Omega_{t}$, the growth rate is $g_{t}(\psi, \varphi)=$ $\lambda \sigma l(\psi, \varphi)\left(p_{s}+p_{m} \theta\right)+n$ and the measure of scale effects $\mathcal{S}_{t}$ is nil. There are no scale effects because there are no GPTs $\left(p_{G}=0\right)$ and because the expanding effect and the specialization effect compensate each other $\left(\theta\left(L_{t}\right)=\theta\right)$.

Proof. The proof is straightforward. Plugging $p_{G}=0, p_{m}>0$ and $\theta\left(L_{t}\right)=\theta, \theta>1$ in (6), one has $\mathbb{E}\left[\Theta_{\omega t}\right] \equiv \mathbb{E}\left[\Theta_{t}\right]=p_{m} \theta>0, \forall \omega \in \Omega_{t}$. From (13) and (14), one gets $g_{t}(\psi, \varphi)=$ $\lambda \sigma l(\psi, \varphi)\left(p_{s}+p_{m} \theta\right)+n$ and $\mathcal{S}_{t}=0$, respectively.

This independence may stem from the assumption that (a) spillovers across firms are absent (e.g. Peretto 1999), that (b) spillovers depend on average knowledge (e.g. Smulders \& Van de Klundert 1995; Peretto 1998; Dinopoulos \& Thompson 1998), or that (c) spillovers depend on the knowledge of the most advanced firm (e.g. Young 1998; Aghion \& Howitt 1998; Howitt 1999). All these models have the property that a large economy replicates the structure of a small economy. [...] Moreover, although they allow for spillovers, all these models assume that a larger number of firms undertaking independent R\&D projects does not support a larger aggregate stock of public knowledge. The implicit assumption is that all public knowledge is replicated". 
Proposition 7 shows that, even if some inter-sectoral knowledge diffusion (i.e. knowledge spillovers) exists in the model, it is possible that it does not exhibit scale effects. Let us develop on this issue. In this proposition, knowledge diffusion is such that the intensity of inter-sectoral knowledge diffusion is $\mathbb{E}\left[\Theta_{t}\right]=p_{m} \theta$. The resulting pool of knowledge and law of knowledge accumulation in any sector $\omega$ are $\mathcal{P}_{\omega t}=\mathcal{P}_{t}=\left(p_{s}+p_{m} \theta\right) \chi_{t}$ and $\dot{\chi}_{\omega t}=\dot{\chi}_{t}=\lambda \sigma l(\psi, \varphi) \mathcal{P}_{t}, \forall \omega \in \Omega_{t}$, respectively. Hence, the marginal productivity of labor in $\mathrm{R} \& \mathrm{D}$ is $\frac{\partial \dot{\chi}_{t}}{\partial l_{t}}=\lambda \sigma\left(p_{s}+p_{m} \theta\right) \chi_{t}$. The key point is that, since the pools of knowledge do not depend on the size of the economy $L_{t}$, the marginal productivity of labor in $\mathrm{R} \& \mathrm{D}$ is also independent of $L_{t}$. That is why there are no scale effects.

The Schumpeterian growth framework developed so far in 4.2 is in line with the fully endogenous growth theory as it does not exhibit scale effects and maintains the impact of public policies on the growth rate. Moreover, contrary to the one of 4.1, the framework presented here in 4.2.1 preserves some inter-sectoral knowledge diffusion. However, the assumptions regarding this diffusion can appear questionable. Indeed, Proposition 7 assumes that knowledge diffusion is never expanding: absence of GPTs and restrictions on the way medium knowledge diffuses. In the subsequent subsections, we return to these two assumptions and we relax them.

\subsubsection{What about general purpose technologies?}

Let us assume that $p_{G}>0$. In other words, we now allow for the possible arrival of GPTs (as described above in Subsection 3.1.1- $i$, we thus consider that some knowledge can diffuse to the whole set of sectors). It is clear that this generate scale effects. Are they significant?

In order to focus on the impact of GPTs on scale effects, let us furthermore assume that - as previously in 4.2 .1 - the diffusion of medium knowledge is constant: $\theta\left(L_{t}\right)=\theta$. One gets the following proposition:

Proposition 8. If $p_{G}>0, p_{m} \geq 0$ and $\theta\left(L_{t}\right)=\theta, \theta>1$, then the intensity of inter-sectoral knowledge diffusion is $\mathbb{E}\left[\Theta_{\omega t}\right] \equiv \mathbb{E}\left[\Theta_{t}\right]=p_{G} \gamma L_{t}+p_{m} \theta, \forall \omega \in \Omega_{t}$. The growth rate is $g_{t}(\psi, \varphi)=$ $\lambda \sigma l(\psi, \varphi)\left(p_{s}+p_{G} \gamma L_{t}+p_{m} \theta\right)+n$ and the measure of scale effects, $\mathcal{S}_{t}=\lambda \sigma l(\psi, \varphi) p_{G} \gamma$, is strictly positive. There are scale effects only because of the occurrence of GPTs.

Proof. The proof is straightforward. Rewriting (6), (13) and (14), under the assumptions $p_{G}>0$, $p_{m} \geq 0$ and $\theta\left(L_{t}\right)=\theta, \theta>1$, one gets the above expressions of $\mathbb{E}\left[\Theta_{\omega t}\right], g_{t}(\psi, \varphi)$ and $\mathcal{S}_{t}$.

Proposition 8 clearly shows that the presence of GPTs generates scale effects: their measure $\mathcal{S}_{t}$ is strictly positive and it depends on $p_{G}$, the probability of occurrence of GPTs. Because the existence of GPTs is not questionable, one could think that one faces here a paradox in the sense that GPTs leads to a property of the model (scale effects) which is at odds with well established empirical facts (no significant evidence of scale effects).

In fact, this contradiction is only apparent. Indeed, as emphasized by the literature on GPTs, we know that it is empirically reasonable to assume that, in the large mass of discoveries, GPTs are quite rare. ${ }^{19}$ In the present framework, this amounts to assuming that $p_{G}$ is low. Therefore, even if GPTs create scale effects, their measure $\mathcal{S}_{t}$ is small (moreover, one has $\lim _{P_{G} \rightarrow 0} \mathcal{S}_{t}=0$ ).

The intuitions behind these results are the following. The intensity of inter-sectoral knowledge diffusion is $\mathbb{E}\left[\Theta_{t}\right]=p_{G} \gamma L_{t}+p_{m} \theta$. The resulting pool of knowledge and law of knowledge accumulation in any sector $\omega$ are $\mathcal{P}_{\omega t}=\mathcal{P}_{t}=\left(p_{s}+p_{G} \gamma L_{t}+p_{m} \theta\right) \chi_{t}$ and $\dot{\chi}_{\omega t}=\dot{\chi}_{t}=\lambda \sigma l(\psi, \varphi) \mathcal{P}_{t}, \forall \omega \in \Omega_{t}$, respectively. Hence, the marginal productivity of labor in $\mathrm{R} \& \mathrm{D}$ is $\frac{\partial \dot{\chi}_{t}}{\partial l_{t}}=\lambda \sigma\left(p_{s}+p_{G} \gamma L_{t}+p_{m} \theta\right) \chi_{t}$. Contrary to the previous case analyzed in Subsection 4.2.1, the pools of knowledge now depend on the size of the economy $L_{t}$, and so does the marginal productivity of labor in R\&D. That is why there are scale effects. However, the key point here lies in that, since the probability $p_{G}$ is low, the impact of $L_{t}$ on the marginal productivity is weak, and thus scale effects are not significant.

To sum up, allowing the occurrence of GPTs in the present framework does not necessarily contradict empirical findings along which there is no evidence of the presence of scale effects.

\footnotetext{
${ }^{19}$ See, for instance the essays included in Helpman (1998).
} 


\subsubsection{Size of the economy, expanding knowledge diffusion, complexity and specializ- ation}

In 4.2.1 and 4.2.2, the diffusion of medium knowledge was assumed to be independent of the size of the economy $L_{t}$ (i.e. the expanding and specialization effects were neutralizing each other). Now, we relax this simplifying assumption by considering that medium knowledge diffuses on a subset of sectors of measure $\theta_{t}$, which is function of $N_{t}=\gamma L_{t}$, and we study how this affects scale effects. More particularly, we aim to investigate under which reasonable assumptions on the diffusion of medium knowledge, one can obtain a Schumpeterian growth model in adequation with empirical evidences along which there are no (significant) scale effects.

In order to focus on the part played by medium knowledge diffusion, we assume that there are no GPTs (their impact on scale effects has been previously studied in 4.2.2) and we consider more general assumptions on how inter-sectoral knowledge diffusion expands, or contracts, when the size of the economy, $L_{t}$, increases (i.e. we return to the general case of diffusion of medium knowledge presented above in Subsection 3.1.1-iii). Formally, we assume $p_{G}=0$ and $\theta_{t}=\theta\left(L_{t}\right)$. Accordingly, from (6), (13) and (14), the intensity of inter-sectoral knowledge diffusion is $\mathbb{E}\left[\Theta_{\omega t}\right] \equiv$ $\mathbb{E}\left[\Theta_{t}\right]=p_{m} \theta\left(L_{t}\right), \forall \omega \in \Omega_{t}$, the growth rate is $g_{t}(\psi, \varphi)=\lambda \sigma l(\psi, \varphi)\left[p_{s}+p_{m} \theta\left(L_{t}\right)\right]+n$ and the measure of scale effects is $\mathcal{S}_{t}=\lambda \sigma l(\psi, \varphi) p_{m} \theta^{\prime}\left(L_{t}\right)$.

Clearly, $\mathcal{S}_{t}$ - which measures the impact of $L_{t}$ on $g_{t}(\psi, \varphi)$ - depends on the sign of the derivative $\theta^{\prime}\left(L_{t}\right)$, that is of the relative significance of the expanding and specialization effects. The case in which the expanding effect overcomes the specialization one $\left(\theta^{\prime}\left(L_{t}\right)>0\right)$ is analyzed in Proposition 9 , while the case in which the specialization effect overcomes the expanding one $\left(\theta^{\prime}\left(L_{t}\right)<0\right)$ is studied in Proposition 10.

\section{Expanding diffusion of medium knowledge $\left(\theta^{\prime}\left(L_{t}\right)>0\right)$ and complexity.}

Proposition 9. Assume $p_{G}=0, p_{m}>0$, and $\theta_{t}=\theta\left(L_{t}\right)$, where $\theta($.$) is a function of class C^{2}$. If $\theta^{\prime}\left(L_{t}\right)>0$, then the measure of scale effects $\mathcal{S}_{t}$ is positive. There are scale effects only because the expanding effect overcomes the specialization effect $\left(\theta^{\prime}\left(L_{t}\right)>0\right)$.

In this proposition, scale effects result not from the presence of GPTs $\left(p_{G}\right.$ is assumed to be nil), but only from the fact that medium knowledge diffusion expands with $L_{t}$ (i.e. $\theta^{\prime}\left(L_{t}\right)>0$ ). However, as shown in the following corollary, adding some extra assumptions on the function $\theta\left(L_{t}\right)$ allows us to mitigate the empirical and theoretical issues involved by the presence of scale effects while maintaining expanding diffusion.

Corollary. Introducing a "complexity effect".

a) If $\theta^{\prime \prime}\left(L_{t}\right)<0$, then $\dot{\mathcal{S}}_{t}<0, \forall t$ : the measure of scale effects is decreasing over time.

b) If $\lim _{L_{t} \rightarrow \infty} \theta^{\prime}\left(L_{t}\right)=0,{ }^{20}$ then $\lim _{t \rightarrow \infty} S_{t}=0$ : scale effects asymptotically vanish.

This corollary basically shows that the non desirable property of scale effects can be alleviated by assuming that the speed at which medium knowledge diffusion expands is at some point lower than the one at which the size of the economy increases. Within our framework with inter-sectoral knowledge diffusion, this can correspond to the fact that the speed at which the scope of knowledge diffusion expands is curbed down by the proliferation of sectors $\left(\theta\left(L_{t}\right)\right.$ assumed concave, as in point a), or/and to the fact that this scope becomes asymptotically constant $\left(\lim _{L_{t} \rightarrow \infty} \theta^{\prime}\left(L_{t}\right)=0\right.$, as in point b).

To sum up, Proposition 9 and its corollary show that, even if there are scale effects induced by the fact that the expanding effect overcomes the specialization effect $\left(\theta^{\prime}\left(L_{t}\right)>0\right)$, they can be mitigated by the introduction of a "complexity effect" $\left(\theta^{\prime \prime}\left(L_{t}\right)<0\right.$ or/and $\left.\lim _{L_{t} \rightarrow \infty} \theta^{\prime}\left(L_{t}\right)=0\right)$, allowing us to match empirical evidences and to have a non explosive growth rate. Here, the size of the subset of sectors that are impacted by sectors producing medium knowledge increases $\left(\theta^{\prime}\left(L_{t}\right)>0\right)$ but less than the size of the economy; in other words, knowledge diffusion expands, but it is slowed down by increasing complexity.

\footnotetext{
${ }^{20}$ In fact, this limit case corresponds to the case presented in 4.2.1 (see Proposition 7 ) in which it had been assumed that $\theta\left(L_{t}\right)=\theta$.
} 


\section{Contracting diffusion of medium knowledge $\left(\theta^{\prime}\left(L_{t}\right)<0\right)$ : specialization.}

Proposition 10. Assume $p_{G}=0, p_{m}>0$, and $\theta_{t}=\theta\left(L_{t}\right)$, where $\theta($.$) is a function of class C^{2}$. If $\theta^{\prime}\left(L_{t}\right)<0$, then the measure of scale effects $\mathcal{S}_{t}$ is negative. Here, the growth rate decreases as the size of the economy increases because the specialization effect overcomes the expanding effect $\left(\theta^{\prime}\left(L_{t}\right)<0\right)$.

This proposition exhibits a result along which the growth rate of the economy is impacted by the size of the economy, but negatively. The growth rate decreases as the size of the economy increases due to the fact that the expanding effect is outweighed by the specialization effect. Why is it so? In fact, from Assumption 1, we know that the number of sectors increases as the population level increases. Furthermore, even if this framework considers inter-sectoral knowledge diffusion, specialization $\left(\right.$ i.e. $\left.\theta^{\prime}\left(L_{t}\right)<0\right)$ implies that sectors are less likely to interact: here, the diffusion of knowledge tends to contract.

\section{The intuitions behind the results obtained in Propositions 9 and 10 are the following.}

The pool of knowledge and the law of knowledge accumulation in any sector $\omega, \omega \in \Omega_{t}$, are $\mathcal{P}_{\omega t}=\mathcal{P}_{t}=\left[p_{s}+p_{m} \theta\left(L_{t}\right)\right] \chi_{t}$ and $\dot{\chi}_{\omega t}=\dot{\chi}_{t}=\lambda \sigma l(\psi, \varphi) \mathcal{P}_{t}$, respectively. Therefore, the marginal productivity of labor in R\&D activity is $\frac{\partial \dot{\chi}_{t}}{\partial l_{t}}=\lambda \sigma\left[p_{s}+p_{m} \theta\left(L_{t}\right)\right] \chi_{t}$. Here, the pools of knowledge, and thus the marginal productivity of labor in $R \& D$, depend on the size of the economy $L_{t}$. This impact is unclear since one has ${ }^{21}$

$$
\frac{\partial}{\partial L_{t}}\left(\frac{\partial \dot{\chi}_{t}}{\partial l_{t}}\right)=\lambda \sigma\left[1+g_{\chi_{t}}(\psi, \varphi)\right] p_{m} \theta^{\prime}\left(L_{t}\right) \chi_{t}
$$

In Proposition 9, it is assumed that $\theta^{\prime}\left(L_{t}\right)$ is positive. Then the marginal productivity of labor in $R \& D$ increases with the size of the economy, since $\frac{\partial}{\partial L_{t}}\left(\frac{\partial \dot{\chi}_{t}}{\partial l_{t}}\right)>0$. Here, since the expanding effect overcomes the specialization one, when the number of sector increases, each sector interacts with an increasing number of sectors. Hence, as the size of the economy increases, the size of the pools of knowledge, and thus the marginal productivity of labor in $R \& D$, increase. That is why there are scale effects. However, one can mitigate these effects by making some extra assumptions on $\theta($.$) . If \theta^{\prime \prime}\left(L_{t}\right)$ is assumed to be negative (i.e. if the diffusion of knowledge does not expand as fast as the size of the economy), then the measure of scale effects decreases over time $\left(\dot{\mathcal{S}}_{t}<0\right)$. This results from the fact that the marginal productivity of labor in R\&D does not increase as fast as the size of the economy: increasing complexity somehow dilutes scale effects. If it is assumed that $\lim _{L_{t} \rightarrow \infty} \theta^{\prime}\left(L_{t}\right)=0$, then scale effects asymptotically vanish because the marginal productivity of labor in R\&D increases less and less as the size of the economy increases, and eventually becomes constant asymptotically: increasing complexity suppresses scale effects.

Basically, the underlying idea consists in assuming that, as the size of the economy (and thus the number of sectors) increases, the knowledge produced in each sector impacts a growing number of sectors (expanding knowledge diffusion). However, complexity implies that the proportion of sectors impacted within the economy decreases and eventually tends to zero. This explains the presence of scale effects, but also why they may progressively vanish as the size of the economy increases.

In Proposition 10, it is assumed that $\theta^{\prime}\left(L_{t}\right)$ is negative. Then the marginal productivity of labor in R\&D decreases with the size of the economy, since $\frac{\partial}{\partial L_{t}}\left(\frac{\partial \dot{\chi}_{t}}{\partial l_{t}}\right)<0$. Here, the specialization effect overcomes the expanding one. Accordingly, when the number of sector increases, each sector is likely to interact with a decreasing number of sectors (specialization effect). Hence, as the size of the economy increases, the size of the pools of knowledge, and thus the marginal productivity

${ }^{21}$ The impact of $L_{t}$ on the marginal productivity of labor in R\&D is given by

$$
\frac{\partial}{\partial L_{t}}\left(\frac{\partial \dot{\chi}_{t}}{\partial l_{t}}\right)=\lambda \sigma \frac{\partial}{\partial L_{t}}\left(\left[p_{s}+p_{m} \theta\left(L_{t}\right)\right] \chi_{t}\right)=\lambda \sigma\left[p_{m} \theta^{\prime}\left(L_{t}\right) \chi_{t}+\left[p_{s}+p_{m} \theta\left(L_{t}\right)\right] \frac{\partial \chi_{t}}{\partial L_{t}}\right]
$$

Furthermore, one has $\frac{\partial \chi_{t}}{\partial L_{t}}=\frac{\partial}{\partial L_{t}}\left(\chi_{0} e^{\lambda \sigma l(\psi, \varphi)\left(p_{s}+p_{m} \theta\left(L_{t}\right)\right)}\right)=\lambda \sigma l(\psi, \varphi) p_{m} \theta^{\prime}\left(L_{t}\right) \chi_{t}$. One obtains $\frac{\partial}{\partial L_{t}}\left(\frac{\partial \dot{\chi}_{t}}{\partial l_{t}}\right)=$ $\lambda \sigma\left\{1+\left[p_{s}+p_{m} \theta\left(L_{t}\right)\right] \lambda \sigma l(\psi, \varphi)\right\} p_{m} \theta^{\prime}\left(L_{t}\right) \chi_{t}$. Since $g_{\chi_{t}}(\psi, \varphi)=\left[p_{s}+p_{m} \theta\left(L_{t}\right)\right] \lambda \sigma l(\psi, \varphi)$, one gets $(16)$. 
of labor in R\&D, decrease. ${ }^{22}$ That is why this framework exhibits negative scale effects. ${ }^{23}$

\subsubsection{Why the presence of inter-sectoral knowledge diffusion and the absence of scale effects can be compatible: main results}

The previous analysis enabled us to obtain a Schumpeterian growth model that does not exhibit scale effects (or at least not significant ones), in which public policies have an impact on the growth rate, in which GPTs can occur, and in which medium knowledge diffusion may be affected by the fact that the size of the economy increases. In order to comply with these commonly agreed empirical facts, we specified several reasonable assumptions on inter-sectoral knowledge diffusion: namely on the occurrence of GPTs (i.e. on the probability $p_{G}$ ) and on the way medium knowledge diffuses (i.e. the impact of the size of the economy $L_{t}$ on the diffusion of medium knowledge, that is on the shape of the function $\theta\left(L_{t}\right)$ ). In Proposition 11 below, we recapitulate the main results regarding the link between knowledge diffusion and scale effects. Most of these results have been proven in the propositions previously presented in the paper. In particular, we have isolated the effects of GPTs (4.2.2) and of the diffusion of medium knowledge (4.2.3); these two effects can now be considered together (see points 3 and 4 of the proposition). Finally, following Peretto \& Smulders (2002), we will conclude our analysis by defining a concept technological distance that we will use to reinterpret our main results.

Proposition 11. If $p_{G} \geq 0, p_{m} \geq 0$, and $\theta_{t}=\theta\left(L_{t}\right)$, where $\theta($.$) is a function of class C^{2}$, then the scope of knowledge diffusion is $\mathbb{E}\left[\Theta_{t}\right]=p_{G} \gamma L_{t}+p_{m} \theta\left(L_{t}\right)$, the growth rate is $g_{t}(\psi, \varphi)=$ $\lambda \sigma l(\psi, \varphi)\left(p_{s}+\mathbb{E}\left[\Theta_{t}\right]\right)+n$, and the measure of scale effects is $\mathcal{S}_{t}=\lambda \sigma l(\psi, \varphi)\left[p_{G} \gamma+p_{m} \theta^{\prime}\left(L_{t}\right)\right]$.

1. If $p_{G}=p_{m}=0$ (i.e. $p_{s}=1$ ), then $\mathbb{E}\left[\Theta_{t}\right]=0$ (i.e. there is no inter-sectoral knowledge diffusion). Therefore, $\mathcal{S}_{t}=0$ (i.e. there are no scale effects) due to the absence of intersectoral knowledge diffusion.

2. If $p_{G}=0, p_{m}>0$ and $\theta\left(L_{t}\right)=\theta>0$ (the expanding effect and the specialization effect compensate each other: $\left.\theta^{\prime}\left(L_{t}\right)=0\right)$, then $\mathbb{E}\left[\Theta_{t}\right]=p_{m} \theta>0$ and $\mathcal{S}_{t}=0$. In spite of the fact that there is some inter-sectoral knowledge diffusion, there are no scale effects.

3. If $p_{G}>0, p_{m}>0$ and $\theta_{t}=\theta\left(L_{t}\right)$ with $\theta^{\prime}\left(L_{t}\right)>0$ (the expanding effect overcomes the specialization effect), then $\mathbb{E}\left[\Theta_{t}\right]=p_{G} \gamma L_{t}+p_{m} \theta\left(L_{t}\right)>0$ and $\mathcal{S}_{t}>0$. Due to both GPTs and expanding diffusion of medium knowledge, there are scale effects. However, these scale effects are not necessarily a problem:

- The impact of GPTs can be considered as weak, since $p_{G}$ can be assumed small.

- The impact of expanding medium knowledge diffusion can be mitigated by introducing a complexity effect by assuming $\theta^{\prime \prime}\left(L_{t}\right)<0$ and/or $\lim _{L_{t} \rightarrow \infty} \theta^{\prime}\left(L_{t}\right)=0$. Such a complexity effects implies that scale effects diminish over time $\left(\dot{\mathcal{S}}_{t}<0\right)$ and/or they asymptotically vanish $\left(\lim _{t \rightarrow \infty} S_{t}=0\right)$.

4. If $p_{G}>0, p_{m}>0$ and $\theta_{t}=\theta\left(L_{t}\right)$ with $\theta^{\prime}\left(L_{t}\right)<0$ (the specialization effect overcomes the expanding effect), then $\mathbb{E}\left[\Theta_{t}\right]=p_{G} \gamma L_{t}+p_{m} \theta\left(L_{t}\right)>0$ and $\mathcal{S}_{t} \gtreqless 0$. The scale effects generated by GPTs may be more of less offset by the specialization that contracts the diffusion of medium knowledge.

We now provide some comments on each of the points of Proposition 11.

The first point shows that the absence of inter-sectoral knowledge diffusion $\left(\mathbb{E}\left[\Theta_{t}\right]=0\right.$, or equivalently $p_{G}=p_{m}=0$ ) is a sufficient condition in order to eliminate scale effects (this result was established in Proposition 6).

\footnotetext{
${ }^{22}$ Here, the size of the pools decreases because, in this paper, knowledge diffusion formalization lies on the fact that sectors share a more or less large number of stocks of knowledge, each of which being produced by a given sector. Specialization implies that each sector interacts with fewer sectors as the number of sectors increases. Hence, each sector will be able to use fewer stocks; that is why its pool of knowledge decreases with the size of the economy. One could adapt the formalization to consider that it is the flow of knowledge inherent in each innovation that diffuses and not the accumulated stock. On the distinction between these flows and stocks of knowledge, see Gray \& Grimaud (2016).

${ }^{23}$ Jones (1999) obtains negative scale effects by using the assumption along which "the number of sectors grows less than proportionally with population".
} 
However, as seen in the second point (which summarizes the results of Proposition 7), it is not a necessary condition to have fully endogenous growth model that does not exhibit scale effects. In fact, there is no contradiction between the presence of inter-sectoral knowledge diffusion and the absence of scale effects. Indeed, we have shown that a sufficient condition not to have scale effects is that the scope of knowledge diffusion $\mathrm{E}\left[\Theta_{t}\right]$ is independent of the size of the economy. In our framework, this occurs only in the case in which there are no GPTs $\left(p_{G}=0\right)$ and there is medium knowledge diffusion $\left(p_{m}>0\right)$ such that the expanding and specialization effects exactly balance each others $\left(\theta\left(L_{t}\right)=\theta\right)$.

In the third and fourth points of Proposition 11, we investigate whether one can relax these two restrictions on the way the knowledge created in some sectors may impact other sectors R\&D activities. Regarding GPTs, we have shown in Proposition 8 that they undoubtedly generate scale effects. Nevertheless, since the occurrence of GPTs is rare, it is reasonable to state that these scale effects are not significant. Therefore, the presence of GPTs does not lead to a model which would contradict empirical observations regarding the absence of scale effects. Regarding medium knowledge diffusion, it could be considered that the expanding and specialization effects do not perfectly balance. Accordingly, the scope of knowledge diffusion, $\mathbb{E}\left[\Theta_{t}\right]$, would depend on the size of the economy not only because of the presence of GPTs, but also because the scope of diffusion of medium knowledge, $\theta\left(L_{t}\right)$, is not independent of the size of the economy $L_{t}$. In the third point, we extend the results of Proposition 9 and of its corollary by considering simultaneously the presence of GPTs and the fact that the expanding effect overcomes the specialization one $\left(\theta^{\prime}\left(L_{t}\right)>0\right)$. Despite the fact that, because $\mathbb{E}\left[\Theta_{t}\right]$ is increasing in $L_{t}$, the model exhibits the non desirable property of scale effects $\left(\mathcal{S}_{t}>0\right)$, it can still be in accordance with empirical evidence on this issue. First, the impact of GPTs on scale effects is negligible. Second, the impact of expanding medium knowledge diffusion on scale effects is reduced under the assumption of the presence of a "complexity effect" $\left(\theta^{\prime \prime}\left(L_{t}\right)<0\right.$ and /or $\left.\lim _{L_{t} \rightarrow \infty} \theta^{\prime}\left(L_{t}\right)=0\right)$. In the fourth point, we extend the result of Proposition 10: it appears that - because the specialization effect overcomes the expanding one $\left(\theta^{\prime}\left(L_{t}\right)<0\right)$ - the measure of scale effects $\mathcal{S}_{t}$ may be positive, nil, or even negative, depending on the sign of $p_{G} \gamma+p_{m} \theta^{\prime}\left(L_{t}\right)$. If $p_{G}>-\frac{p_{m}}{\gamma} \theta^{\prime}\left(L_{t}\right)$, it is positive. But, as argued above in the comments of the third point, there is no real problem regarding the scale effects issue. Besides, it is interesting to note that, when the presence of GPTs is combined with the case in which the specialization effect overcomes the expanding effect, one can obtain a model in which there are negative scale effects. Indeed, the positive effect of the population size on the growth rate induced by the presence of GPTs can be offset by the negative effect induced by the fact that specialization somehow dilutes (contracts) the subset of sectors on which medium knowledge diffuses.

\section{Remark: technological distance}

Let us come back to the concept of technological distance mentioned above in Subsection 3.1. As argued by Peretto \& Smulders (2002), when the technological distance between the creators and the users of knowledge increases, firms are less likely to be able to benefit from the knowledge created by other firms. In our framework, the wider the expected scope of knowledge diffusion, $\mathbb{E}\left[\Theta_{\omega t}\right]$, the more likely firms are to interact. Accordingly, it is reasonable to consider that the technological distance should decrease with $\mathbb{E}\left[\Theta_{t}\right]$. We thus propose to define the technological distance, that we denote by $\mathcal{D}_{t}$, as follows:

$$
\mathcal{D}_{t}=\frac{1}{\mathbb{E}\left[\Theta_{t}\right]}=\frac{1}{p_{G} \gamma L_{t}+p_{m} \theta\left(L_{t}\right)} \in[0 ; \infty)
$$

All the previous results can be reinterpreted using the concept of technological distance, which measures the significance of interactions between sectors' $R \& D$ activities. The main results on the link between scale effects and knowledge diffusion were presented in Proposition 11. In its corollary, we present the link between scale effects and technological distance. ${ }^{24}$

\footnotetext{
${ }^{24}$ This definition considers the technological distance between a given sector $\omega$ and the sectors from which it receives knowledge, that one could name its network. This sector $\omega$ 's network comprises all sectors producing GPTs (see the term $p_{G} \gamma L_{t}$ in (17)) and sectors in the neighborhood of $\omega,\left[\omega-\theta\left(L_{t}\right) / 2 ; \omega+\theta\left(L_{t}\right) / 2\right]$, on the Salop circle (see the term $p_{m} \theta\left(L_{t}\right)$ in (17)). The first and second derivatives of $\mathcal{D}_{t}$ are respectively

$$
\frac{\partial \mathcal{D}_{t}}{\partial L_{t}}=-\frac{p_{G} \gamma+p_{m} \theta^{\prime}\left(L_{t}\right)}{\left[p_{G} \gamma L_{t}+p_{m} \theta\left(L_{t}\right)\right]^{2}} \text { and } \frac{\partial^{2} \mathcal{D}_{t}}{\partial L_{t}^{2}}=\frac{2\left[p_{G} \gamma+p_{m} \theta^{\prime}\left(L_{t}\right)\right]^{2}-p_{m} \theta^{\prime \prime}\left(L_{t}\right)\left[p_{G} \gamma L_{t}+p_{m} \theta\left(L_{t}\right)\right]}{\left[p_{G} \gamma L_{t}+p_{m} \theta\left(L_{t}\right)\right]^{3}}
$$
}




\section{Corollary. Scale effects and technological distance.}

1. If $p_{G}=p_{m}=0$ (i.e. $p_{s}=1$ ), then the technological distance $\mathcal{D}_{t}$ is infinite. Because there are no interactions between sectors, there are no scale effects $\left(\mathcal{S}_{t}=0\right)$.

2. If $p_{G}=0, p_{m}>0$ and $\theta\left(L_{t}\right)=\theta>0$, then the technological distance is constant $\left(\mathcal{D}_{t}=\right.$ $\left.1 / p_{m} \theta\right)$. As the size of the economy increases, the significance of interactions between sectors remains unchanged. That is why there are no scale effects $\left(\mathcal{S}_{t}=0\right)$.

3. If $p_{G}>0, p_{m}>0$ and $\theta_{t}=\theta\left(L_{t}\right)$ with $\theta^{\prime}\left(L_{t}\right)>0$, then $\frac{\partial \mathcal{D}_{t}}{\partial L_{t}}<0$. The technological distance decreases with the size of the economy for two reasons: the presence of GPTs and the fact that the expanding effect overcomes the specialization effect (i.e. $\theta^{\prime}\left(L_{t}\right)>0$ ). Because this leads to more interactions between sectors, there are scale effects $\left(\mathcal{S}_{t}>0\right)$.

4. If $p_{G}>0, p_{m}>0$ and $\theta_{t}=\theta\left(L_{t}\right)$ with $\theta^{\prime}\left(L_{t}\right)<0$, then $\frac{\partial \mathcal{D}_{t}}{\partial L_{t}} \gtreqless 0$. The technological distance can decrease or increase with the size of the economy. Indeed, the presence of GPTs tends to make $\mathcal{D}_{t}$ decreasing; conversely, the fact that the specialization effect overcomes the expanding effect (i.e. $\left.\theta^{\prime}\left(L_{t}\right)<0\right)$ tends to make $\mathcal{D}_{t}$ increasing. Consequently, there might be scale effects or not, and even negative scale effects $\left(\mathcal{S}_{t} \gtreqless 0\right)$.

Peretto \& Smulders (2002) present an endogenous growth model in which they "posit a mechanism whereby technological distance increases with the size of the economy". Their assumption corresponds to the ones considered in our point 4 when $\frac{\partial \mathcal{D}_{t}}{\partial L_{t}}>0$, which occurs if the specialization effect overcomes not only the expanding effect $\left(\right.$ i.e. $\left.\theta^{\prime}\left(L_{t}\right)<0\right)$ but also the presence of GPTs (i.e. $\left.\theta^{\prime}\left(L_{t}\right)<-\frac{p_{G} \gamma}{p_{m}}\right)$. These types of assumptions lead to models that can exhibit positive or negative scale effects.

Besides, in Peretto \& Smulders (2002), scale effects always vanish asymptotically because the occurrence of new lines of research ("accumulation along the extensive margin") "leads to higher specialization in firms' R\&D activities and to dilution of spillovers". We obtain a similar result in our point 3 even if, contrary to their assumption, we assume that the technological decreases with the size of the economy. In this case, assuming $\theta^{\prime \prime}\left(L_{t}\right)<0$, one gets $\frac{\partial^{2} \mathcal{D}_{t}}{\partial L_{t}^{2}}>0$ : because the technological distance decreases less quickly than the size of the economy increases, scale effects diminish over time $\left(\dot{\mathcal{S}}_{t}<0\right)$. Furthermore, if one assume that $p_{G}$ tends to zero and that $\lim _{L_{t} \rightarrow \infty} \theta^{\prime}\left(L_{t}\right)=0$, then $\lim _{L_{t} \rightarrow \infty} \frac{\partial \mathcal{D}_{t}}{\partial L_{t}}=0$ : because the technological distance becomes constant asymptotically, scale effects asymptotically vanish $\left(\lim _{t \rightarrow \infty} S_{t}=0\right)$. This limit case of point 3 is in fact the point 2 .

\section{Conclusion}

This paper develops the idea that there is a close link between the fact that fully endogenous growth models exhibit (or not) the non desirable scale effects property and the intensity of knowledge diffusion considered in such models.

This link clearly appears at first when studying two polar cases of Schumpeterian models. On one end, a model assuming knowledge spillovers across all sectors (i.e. considering that the R\&D activity of each sector uses a pool of knowledge that comprises all the knowledge accumulated in the economy) exhibits scale effects. On the other end, a model assuming no inter-sectoral knowledge spillovers (i.e. considering that the $\mathrm{R} \& \mathrm{D}$ activity of each sector uses a pool that consists only of the knowledge accumulated so far within this sector) does not display scale effects. The underlying reason of the link between scale effects and knowledge diffusion is found in the impact of the pools of knowledge on the marginal productivity of labor in R\&D. The basic insights are as follows. The wider inter-sectoral knowledge diffusion, the larger the pool of knowledge used by each sector's R\&D activity. Then, the more knowledge diffusion spreads with the size of the economy (as measured equivalently by the number of sectors or by the population level), the more likely an increase in the size of the economy will lead to larger pools of knowledge, implying a higher marginal productivity of labor in R\&D activity, more innovations, and thus a higher growth rate. Hence, the more 
knowledge diffusion spreads along with the size of the economy, the more likely the model will display scale effects.

Accordingly, it becomes obvious that a sufficient condition to have a scale-invariant fully endogenous growth model is to assume no inter-sectoral knowledge diffusion. In fact, this has been the main channel used in the literature to remove scale effects while maintaining the effect of R\&D policies on the growth rate. However, the point is that the assumption along which there would be no inter-sectoral knowledge spillovers is not reasonable, neither theoretically nor empirically. Indeed, many papers have emphasized that knowledge produced in a given sector may be used by the R\&D activities of other sectors (see, for instance, Romer 1990; Scotchmer 1991; Griliches 1992, 1995; Aghion \& Howitt 1998; Weitzman 1998; Hall 2004; Jones 2005; Hall, Mairesse \& Mohnen 2010). Besides, as detailed for instance in Bresnahan \& Trajtenberg (1995) or in Helpman (1998), the occurrence of general-purpose technologies (GPTs) seems indubitable.

We therefore face the following theoretical paradox: there is (some) knowledge diffusion across sectors - including the one resulting from GPTs - but there are no scale effects (or at least they are not empirically significant) and, at first glance, knowledge diffusion implies scale effects.

In this paper, we investigate whether this paradox can be overcome and we show that it is in fact only apparent. In that respect, we use a new methodology: we introduce explicitly knowledge diffusion over a Salop (1979) circle in a standard Schumpeterian growth model; in particular, the formalization we provide explains how knowledge diffusion shapes the pools of knowledge used by $R \& D$ activities, and thus determines the significance of scale effects. The first basic result we obtain within this framework confirms that if there is no inter-sectoral knowledge diffusion, there are no scale effects, precisely because the size of the pools is minimum.

Then, this leads us to tackle the aforementioned paradox. We first build a fully endogenous growth model in which scale effects are cancelled while maintaining some inter-sectoral knowledge diffusion. Even if it solves the paradox, this model is somehow restrictive on the way knowledge diffusion is considered: it does not allow for the occurrence of GPTs and moreover the scope of diffusion of knowledge is not impacted by the size of the economy. Then, we isolate the impact of GPTs on scale effects and we show that, even if a model that considers the possible arrival of GPTs displays scale effects, these effects are not significant since their strength depends on the probability of occurrence of GPTs which is obviously low. Finally, we introduce more general assumptions on how knowledge diffusion expands (or contracts) when the size of the economy increases and we determine under which sets of assumptions one can obtain Schumpeterian growth models that comply with most of the commonly agreed empirical facts - namely the absence of significant scale effects, the impact of public policies on the growth rate, and somehow realistic interactions among sectors R\&D activities (including the occurrence of GPTs).

\section{Appendix}

\subsection{Law of knowledge accumulation - Proof of Proposition 1}

Consider any given sector $\omega, \omega \in \Omega_{t}$, and a time interval $(t, t+\Delta t)$. The level of knowledge in this sector at date $t$ is $\chi_{\omega t}$. Let $k, k \in \mathbb{N}$, be the number of innovations that occur during the interval $(t, t+\Delta t)$. Accordingly, under Assumptions 2 and 3, the level of knowledge at date $t+\Delta t$, $\chi_{\omega t+\Delta t}$, is a random variable taking the values $\left\{\chi_{\omega t}+k \sigma \mathcal{P}_{\omega t}\right\}_{k \in \mathbb{N}}$ with associated probabilities $\left\{\frac{\left(\int_{t}^{t+\Delta t} \lambda l_{\omega u} d u\right)^{k}}{k !} e^{-\int_{t}^{t+\Delta t} \lambda l_{\omega u} d u}\right\}_{k \in \mathbb{N}}$.

Then, the expected level of knowledge at date $t+\Delta t$ is

$$
\begin{aligned}
& \mathbb{E}\left[\chi_{\omega t+\Delta t}\right]=\sum_{k=0}^{\infty} \frac{\left(\int_{t}^{t+\Delta t} \lambda l_{\omega u} d u\right)^{k}}{k !} e^{-\int_{t}^{t+\Delta t} \lambda l_{\omega u} d u}\left[\chi_{\omega t}+k \sigma \mathcal{P}_{\omega t}\right] \\
& =\left[\chi_{\omega t} \sum_{k=0}^{\infty} \frac{\left(\int_{t}^{t+\Delta t} \lambda l_{\omega u} d u\right)^{k}}{k !}+\sigma \mathcal{P}_{\omega t}\left(\int_{t}^{t+\Delta t} \lambda l_{\omega u} d u\right) \sum_{k=1}^{\infty} \frac{\left(\int_{t}^{t+\Delta t} \lambda l_{\omega u} d u\right)^{k-1}}{(k-1) !}\right] e^{-\int_{t}^{t+\Delta t} \lambda l_{\omega u} d u}
\end{aligned}
$$


As $K \rightarrow \infty$, the MacLaurin series $\sum_{k=0}^{K} \frac{\left(\int_{t}^{t+\Delta t} \lambda l_{\omega u} d u\right)^{k}}{k !}$ converges to $e^{\int_{t}^{t+\Delta t} \lambda l_{\omega u} d u}$. One gets

$$
\begin{gathered}
\mathbb{E}\left[\chi_{\omega t+\Delta t}\right]=\left[\chi_{\omega t} e^{\int_{t}^{t+\Delta t} \lambda l_{\omega u} d u}+\sigma \mathcal{P}_{\omega t}\left(\int_{t}^{t+\Delta t} \lambda l_{\omega u} d u\right) e^{\int_{t}^{t+\Delta t} \lambda l_{\omega u} d u}\right] e^{-\int_{t}^{t+\Delta t} \lambda l_{\omega u} d u} \\
\Leftrightarrow \mathbb{E}\left[\chi_{\omega t+\Delta t}\right]=\chi_{\omega t}+\lambda \sigma\left(\int_{t}^{t+\Delta t} l_{\omega u} d u\right) \mathcal{P}_{\omega t}
\end{gathered}
$$

Let $\Lambda_{\omega u}$ denote a primitive of $l_{\omega u}$ with respect to the time variable $u$. Rewriting the previous expression, one exhibits Newton's difference quotients of $\mathbb{E}\left[\chi_{\omega t}\right]$ and of $\Lambda_{\omega t}$ :

$$
\frac{\mathbb{E}\left[\chi_{\omega t+\Delta t}\right]-\chi_{\omega t}}{\Delta t}=\lambda \sigma \frac{\Lambda_{\omega t+\Delta t}-\Lambda_{\omega t}}{\Delta t} \mathcal{P}_{\omega t}
$$

Finally, letting $\Delta t$ tend to zero, one gets $\frac{\partial \mathbb{E}\left[\chi_{\omega t}\right]}{\partial t} \equiv \dot{\chi}_{\omega t}=\lambda \sigma l_{\omega t} \mathcal{P}_{\omega t}$. This proves that the expected knowledge in any sector $\omega$ is a differentiable function of time. Its derivative gives the law of motion of the expected knowledge as given in Proposition 1, in which the expectation operator is dropped to simplify notations.

\subsection{Decentralized economy - Proof of Proposition 4}

Appendix 6.2 provides the detailed analysis of the decentralized economy. In particular, we fully characterize the set of equilibria as functions of the public tools vector $(\psi, \varphi)$. More precisely, we derive the time paths of set of prices and of quantities in the case of constant population growth.

The representative household maximizes her intertemporal utility (8) subject to her budget constraint, $\dot{b}_{t}=w_{t}+r_{t} b_{t}-c_{t}-n b_{t}-T_{t} / L_{t}$, where $b_{t}$ denotes the per capita financial asset and where $T_{t}$ is a lump-sum tax charged by the government in order to finance public policies. This yields the usual Keynes-Ramsey condition:

$$
r_{t}=g_{c_{t}}+\rho
$$

In the final sector, the competitive firm maximizes its profit

$$
\pi_{t}^{Y}=\left(L_{t}^{Y}\right)^{1-\alpha} \int_{\Omega_{t}} \chi_{\omega t}\left(x_{\omega t}\right)^{\alpha} d \omega-w_{t} L_{t}^{Y}-\int_{\Omega_{t}}(1-\psi) q_{\omega t} x_{\omega t} d \omega
$$

The first-order conditions with respect to $L_{t}^{Y}$ and $x_{\omega t}$ give respectively

$$
w_{t}=(1-\alpha) \frac{Y_{t}}{L_{t}^{Y}} \text { and } q_{\omega t}=\frac{\alpha\left(L_{t}^{Y}\right)^{(1-\alpha)} \chi_{\omega t}\left(x_{\omega t}\right)^{\alpha-1}}{1-\psi}, \forall \omega \in \Omega_{t}
$$

Given the production function (11), in each intermediate good sector $\omega, \omega \in \Omega_{t}$, the incumbent monopoly maximizes its profit

$$
\pi_{t}^{x_{\omega}}=q_{\omega t} x_{\omega t}-y_{\omega t}=\left(q_{\omega t}-\chi_{\omega t}\right) x_{\omega t},
$$

where the demand for intermediate $\omega$ is given in (19). The first-order condition with respect to $x_{\omega t}$ gives $\frac{\alpha^{2}\left(L_{t}^{Y}\right)^{(1-\alpha)} \chi_{\omega t}\left(x_{\omega t}\right)^{\alpha-1}}{1-\psi}-\chi_{\omega t}=0, \forall \omega \in \Omega_{t}$.

Hence, one gets the usual result of symmetry in the use of intermediate goods:

$$
x_{\omega t}=x_{t}=\left(\frac{\alpha^{2}}{1-\psi}\right)^{\frac{1}{1-\alpha}} L_{t}^{Y}, \forall \omega \in \Omega_{t}
$$

The final good production function (10) can be rewritten using (21) together with the definition of the whole disposable knowledge in the economy (1); one gets

$$
Y_{t}=\left(\frac{\alpha^{2}}{1-\psi}\right)^{\frac{\alpha}{1-\alpha}} L_{t}^{Y} \mathcal{K}_{t}
$$


Log-differentiating (22) with respect to time gives

$$
g_{Y_{t}}=g_{L_{t}^{Y}}+g_{\mathcal{K}_{t}}
$$

The final good resource constraint (12) can be rewritten using (21) together with (1) and (11); one gets $Y_{t}=L_{t} c_{t}+\left[\alpha^{2} /(1-\psi)\right]^{\frac{1}{1-\alpha}} L_{t}^{Y} \mathcal{K}_{t}$. Dividing both sides by $Y_{t}$ and using (22), one gets $L_{t} c_{t} / Y_{t}=1-\alpha^{2} /(1-\psi)$. Log-differentiating this expression gives $g_{L_{t}}+g_{c_{t}}-g_{Y_{t}}=0$. Since $g_{L_{t}}=n$ from Assumption 1, one gets

$$
g_{Y_{t}}=g_{c_{t}}+n
$$

The wage and the price of intermediate goods given in (19) can be rewritten using (22) and (21), respectively:

$$
w_{t}=(1-\alpha)\left(\frac{\alpha^{2}}{1-\psi}\right)^{\frac{\alpha}{1-\alpha}} \mathcal{K}_{t} \text { and } q_{\omega t}=\frac{\chi_{\omega t}}{\alpha}, \forall \omega \in \Omega_{t}
$$

From (21) and from the marked-up price of intermediate good $\omega$ given in (25), one can rewrite (20), the instantaneous monopoly profit on the sale of each intermediate good $\omega$, as

$$
\pi_{t}^{x_{\omega}}=\frac{1-\alpha}{\alpha}\left(\frac{\alpha^{2}}{1-\psi}\right)^{\frac{1}{1-\alpha}} L_{t}^{Y} \chi_{\omega t}, \forall \omega \in \Omega_{t}
$$

Let us now consider any R\&D activity $\omega, \omega \in \Omega_{t}$, and derive the innovators' arbitrage condition. Given the governmental intervention on behalf of $R \& D$ activities, the incumbent innovator having successfully innovated at date $t$ receives, at any date $\tau>t$, the net profit $\tilde{\pi}_{\tau}^{x_{\omega}}=(1+\varphi) \pi_{\tau}^{x_{\omega}}$ with probability $e^{-\int_{t}^{\tau} \lambda l_{\omega u} d u}$ (i.e. provided that there is no innovation upgrading intermediate good $\omega$ between $t$ and $\tau$ ). The sum of the present values of the incumbent's expected net profits on the sale of intermediate good $\omega$, at date $t$, is therefore $\tilde{\Pi}_{t}^{x_{\omega}}=\int_{t}^{\infty} \tilde{\pi}_{\tau}^{x_{\omega}} e^{-\int_{t}^{\tau}\left(r_{u}+\lambda l_{\omega u}\right) d u} d \tau$. Differentiating this expression with respect to time gives the arbitrage condition in each R\&D activity $\omega$ :

$$
r_{t}+\lambda l_{\omega t}=\frac{\dot{\tilde{\Pi}}_{t}^{x_{\omega}}}{\tilde{\Pi}_{t}^{x_{\omega}}}+\frac{\tilde{\pi}_{t}^{x_{\omega}}}{\tilde{\Pi}_{t}^{x_{\omega}}}, \forall \omega \in \Omega_{t}
$$

The free-entry condition (i.e. zero profit condition) in each R\&D activity $\omega$ is $w_{t}=\lambda \tilde{\Pi}_{t}^{x_{\omega}}$, where $w_{t}$ is the unit cost of labor, given in (25), and where $\lambda \tilde{\Pi}_{t}^{x_{\omega}}$ is the expected revenue when one unit of labor is invested in R\&D. ${ }^{25}$ This gives

$$
\tilde{\Pi}_{t}^{x_{\omega}}=\tilde{\Pi}_{t}^{x}=\frac{1-\alpha}{\lambda}\left(\frac{\alpha^{2}}{1-\psi}\right)^{\frac{\alpha}{1-\alpha}} \mathcal{K}_{t}, \forall \omega \in \Omega_{t}
$$

Log-differentiating (28) with respect to time gives $\dot{\tilde{\Pi}}_{t}^{x_{\omega}} / \tilde{\Pi}_{t}^{x_{\omega}}=g_{\mathcal{K}_{t}}$. Moreover, from (26) and (28), one has

$$
\frac{\tilde{\pi}_{t}^{x_{\omega}}}{\tilde{\Pi}_{t}^{x_{\omega}}}=\frac{(1+\varphi) \frac{1-\alpha}{\alpha}\left(\frac{\alpha^{2}}{1-\psi}\right)^{\frac{1}{1-\alpha}} L_{t}^{Y} \chi_{\omega t}}{\frac{1-\alpha}{\lambda}\left(\frac{\alpha^{2}}{1-\psi}\right)^{\frac{\alpha}{1-\alpha}} \mathcal{K}_{t}}=\frac{(1+\varphi) \lambda \alpha L_{t}^{Y} \chi_{\omega t}}{(1-\psi) \mathcal{K}_{t}}, \forall \omega \in \Omega_{t}
$$

Accordingly, the arbitrage condition (27) writes

$$
r_{t}+\lambda l_{\omega t}=g_{\mathcal{K}_{t}}+\frac{(1+\varphi) \lambda \alpha L_{t}^{Y} \chi_{\omega t}}{(1-\psi) \mathcal{K}_{t}}, \forall \omega \in \Omega_{t}
$$

As usual in the standard literature (see footnote 11), in order to keep the model tractable, we make the standard symmetry assumption, in which $l_{\omega t}=l_{t}$ and $\chi_{\omega t}=\chi_{t}, \forall \omega \in \Omega_{t}$. Under symmetry, one gets the following results:

- The labor constraint (9) (using $N_{t}=\gamma L_{t}$ from Assumption 1) becomes

$$
L_{t}=L_{t}^{Y}+\int_{\Omega_{t}} l_{\omega t} d \omega=L_{t}^{Y}+N_{t} l_{t}=L_{t}^{Y}+\gamma L_{t} l_{t}
$$

\footnotetext{
${ }^{25}$ Indeed, innovations in sector $\omega$ are assumed to occur with a Poisson arrival rate of $\lambda l_{\omega t}$ : for one unit of labor is invested in R\&D activity $\omega$, the probability to obtain one innovation at date $t$ is thus $\lambda$. Moreover, its value, taking into account the R\&D public policy, is $\tilde{\Pi}_{t}^{x_{\omega}}$.
} 
- The whole disposable knowledge (1) becomes $\mathcal{K}_{t}=N_{t} \chi_{t}$. Besides, since one has $N_{t}=\gamma L_{t}$ and $g_{L_{t}}=n$ (Assumption 1), one obtains

$$
g_{\mathcal{K}_{t}}=g_{\chi_{t}}+n
$$

- In any sector $\omega$, the pool of knowledge and the law of knowledge accumulation (Propositions 1 and 3) rewrite $\mathcal{P}_{\omega t}=\mathcal{P}_{t}=\left(p_{s}+\mathbb{E}\left[\Theta_{t}\right]\right) \chi_{t}, \forall \omega \in \Omega_{t}$, and $\dot{\chi}_{\omega t}=\dot{\chi}_{t}=\lambda \sigma l_{t} \mathcal{P}_{t}, \forall \omega \in \Omega_{t}$, respectively. Consequently, the growth rate of knowledge in any sector $\omega$ is

$$
g_{\chi_{\omega t}}=g_{\chi_{t}}=\lambda \sigma\left(p_{s}+\mathbb{E}\left[\Theta_{t}\right]\right) l_{t}, \forall \omega \in \Omega_{t}
$$

- The arbitrage condition in any R\&D activity $\omega, \omega \in \Omega_{t}$, (29) becomes $r_{t}+\lambda l_{t}=g_{\mathcal{K}_{t}}+$ $\frac{(1+\varphi) \lambda \alpha L_{t}^{Y} \chi_{t}}{(1-\psi) N_{t} \chi_{t}}$. Furthermore, using (31), (32) and $N_{t}=\gamma L_{t}$ (Assumption 1), one gets

$$
r_{t}+\lambda l_{t}=\lambda \sigma\left(p_{s}+\mathbb{E}\left[\Theta_{t}\right]\right) l_{t}+n+\frac{(1+\varphi) \lambda \alpha L_{t}^{Y}}{(1-\psi) \gamma L_{t}}
$$

In the following system, we summarize the equations that enable us to characterize the equilibrium:

$$
\left\{\begin{array}{l}
r_{t}=g_{c_{t}}+\rho \\
x_{\omega t}=x_{t}=\left(\frac{\alpha^{2}}{1-\psi}\right)^{\frac{1}{1-\alpha}} L_{t}^{Y}, \forall \omega \in \Omega_{t} \\
Y_{t}=\left(\frac{\alpha^{2}}{1-\psi}\right)^{\frac{\alpha}{1-\alpha}} L_{t}^{Y} \mathcal{K}_{t} \\
g_{Y_{t}}=g_{L_{t}^{Y}}+g_{\mathcal{K}_{t}} \\
g_{Y_{t}}=g_{c_{t}}+n \\
w_{t}=(1-\alpha)\left(\frac{\alpha^{2}}{1-\psi}\right)^{\frac{\alpha}{1-\alpha}} \mathcal{K}_{t} \text { and } q_{\omega t}=\frac{\chi_{\omega t}}{\alpha}, \forall \omega \in \Omega_{t} \\
L_{t}=L_{t}^{Y}+\gamma L_{t} l_{t} \\
g_{\mathcal{K}_{t}}=g_{\chi_{t}}+n \\
g_{\chi_{\omega t}}=g_{\chi_{t}}=\lambda \sigma\left(p_{s}+\mathbb{E}\left[\Theta_{t}\right]\right) l_{t}, \forall \omega \in \Omega_{t} \\
r_{t}+\lambda l_{t}=\lambda \sigma\left(p_{s}+\mathbb{E}\left[\Theta_{t}\right]\right) l_{t}+n+\frac{(1+\varphi) \lambda \alpha L_{t}^{Y}}{(1-\psi) \gamma L_{t}}
\end{array}\right.
$$

From (18) and (33), one gets

$$
g_{c_{t}}+\rho+\lambda l_{t}=\lambda \sigma\left(p_{s}+\mathbb{E}\left[\Theta_{t}\right]\right) l_{t}+n+\frac{(1+\varphi) \lambda \alpha L_{t}^{Y}}{(1-\psi) \gamma L_{t}}
$$

Using (23), (31) and (32), one has $g_{Y_{t}}=g_{L_{t}^{Y}}+g_{\mathcal{K}_{t}}=g_{L_{t}^{Y}}+g_{\chi_{t}}+n=g_{L_{t}^{Y}}+\lambda \sigma\left(p_{s}+\mathbb{E}\left[\Theta_{t}\right]\right) l_{t}+n$. Then, from (24), one obtains

$$
g_{c_{t}}=g_{L_{t}^{Y}}+\lambda \sigma\left(p_{s}+\mathbb{E}\left[\Theta_{t}\right]\right) l_{t}
$$

Combining (34) and (35) gives $g_{L_{t}^{Y}}+\rho+\lambda l_{t}=n+\frac{(1+\varphi) \lambda \alpha L_{t}^{Y}}{(1-\psi) \gamma L_{t}}$. Using (30), and rearranging the terms, one gets

$$
g_{L_{t}^{Y}}-\frac{\lambda}{\gamma L_{t}}\left[1+\frac{1+\varphi}{1-\psi} \alpha\right] L_{t}^{Y}=n-\rho-\frac{\lambda}{\gamma}
$$

In order to solve this differential equation in $L_{t}^{Y}$, we use a variable substitution: let $X_{t}=1 / L_{t}^{Y}$. Log-differentiation with respect to time gives $g_{X_{t}}=-g_{L_{t}^{Y}}$. Substituting into (36) gives the following first-order linear differential equation in $X_{t}$ :

$$
-g_{X_{t}}-\frac{\lambda}{\gamma L_{t}}\left[1+\frac{1+\varphi}{1-\psi} \alpha\right] \frac{1}{X_{t}}=n-\rho-\frac{\lambda}{\gamma} \Leftrightarrow \dot{X}_{t}-\left(\frac{\lambda}{\gamma}+\rho-n\right) X_{t}=-\frac{\lambda}{\gamma}\left[1+\frac{1+\varphi}{1-\psi} \alpha\right] e^{-n t}
$$

Its solution is

$X_{t}=e^{\left(\frac{\lambda}{\gamma}+\rho-n\right) t}\left[X_{0}-\frac{1}{\left(\frac{\lambda}{\gamma}+\rho-n\right)-(-n)} \frac{\lambda}{\gamma}\left(1+\frac{1+\varphi}{1-\psi} \alpha\right)\right]+\frac{1}{\left(\frac{\lambda}{\gamma}+\rho-n\right)-(-n)} \frac{\lambda}{\gamma}\left(1+\frac{1+\varphi}{1-\psi} \alpha\right) e^{-n t}$ 


$$
\Leftrightarrow X_{t}=e^{\left(\frac{\lambda}{\gamma}+\rho-n\right) t}\left[X_{0}-\frac{\lambda}{\lambda+\gamma \rho}\left(1+\frac{1+\varphi}{1-\psi} \alpha\right)\right]+\frac{\lambda}{\lambda+\gamma \rho}\left(1+\frac{1+\varphi}{1-\psi} \alpha\right) e^{-n t}
$$

Accordingly, one gets

$$
L_{t}^{Y}=\frac{\lambda+\gamma \rho}{e^{\left(\frac{\lambda}{\gamma}+\rho-n\right) t}\left[\frac{\lambda+\gamma \rho}{L_{0}^{Y}}-\lambda\left(1+\frac{1+\varphi}{1-\psi} \alpha\right)\right]+\lambda\left(1+\frac{1+\varphi}{1-\psi} \alpha\right) e^{-n t}}
$$

Using the transversality condition in the program of the representative household, one obtains

$$
L_{t}^{Y}(\psi, \varphi)=\frac{1+\frac{\rho \gamma}{\lambda}}{1+\frac{1+\varphi}{1-\psi} \alpha} L_{t}=\frac{(1-\psi)\left(1+\frac{\rho \gamma}{\lambda}\right)}{(1+\varphi) \alpha+1-\psi} L_{t}
$$

Therefore, one has $g_{L_{t}^{Y}}=n$. From (30) and (37), one gets

$$
l_{t}(\psi, \varphi)=\frac{1}{\gamma}-\frac{L_{t}^{Y}}{\gamma L_{t}}=\frac{1}{\gamma}-\frac{1}{\gamma} \frac{(1-\psi)\left(1+\frac{\rho \gamma}{\lambda}\right)}{(1+\varphi) \alpha+1-\psi}=\frac{1}{\gamma}-\frac{\lambda / \gamma+\rho}{\lambda\left(1+\frac{1+\varphi}{1-\psi} \alpha\right)}
$$

The set of Schumpeterian equilibria defined in Definition 1 - i.e. the quantities and prices as functions of the public policy tools vector $(\psi, \varphi)$ - can now be fully characterized. The equilibrium labor partition is given by (37) and (38). From (21) and (37), one gets the equilibrium quantity of each intermediate good $\omega, x_{\omega t}(\psi, \varphi)=x_{t}(\psi, \varphi), \forall \omega \in \Omega_{t}$. From (32) and (38), one gets the growth rate of knowledge in each sector $\omega$ at equilibrium, $g_{\chi_{\omega t}}(\psi, \varphi)=g_{\chi_{t}}(\psi, \varphi)=$ $\lambda \sigma\left(p_{s}+\mathbb{E}\left[\Theta_{t}\right]\right) l(\psi, \varphi), \forall \omega \in \Omega_{t}$. Then, since $g_{L_{t}^{Y}}=n$, the equilibrium growth rate of the economy is $g_{c_{t}}(\psi, \varphi)=g_{Y_{t}}(\psi, \varphi)-n=g_{\mathcal{K}_{t}}(\psi, \varphi)=g_{\chi_{t}}(\psi, \varphi)+n \equiv g_{t}(\psi, \varphi)$. Finally, the equilibrium prices are obtained using (18) and (25) (price of the final good normalized to one). The interest rate is $r_{t}(\psi, \varphi)=g_{t}(\psi, \varphi)+\rho$, the wage is $w_{t}(\psi, \varphi)=(1-\alpha)\left(\frac{\alpha^{2}}{1-\psi}\right)^{\frac{\alpha}{1-\alpha}} \mathcal{K}_{t}(\psi, \varphi)$ and the prices of intermediate goods are $q_{\omega t}(\psi, \varphi)=q_{t}(\psi, \varphi)=\frac{\mathcal{K}_{t}(\psi, \varphi)}{\alpha \gamma L_{t}}, \forall \omega \in \Omega_{t}$, where $\mathcal{K}_{t}(\psi, \varphi)=e^{\int_{0}^{t} g_{s}(\psi, \varphi) d s}$. This proves Proposition 4.

\section{Compliance with ethical standards}

The authors ensure that the researched work submitted is original, fully referenced, exclusive to Journal of Economic Growth, not under consideration in any other journal, not published elsewhere, and that all authors are represented accurately.

Conflict of Interest: The authors declare that they have no conflict of interest.

\section{References}

[1] Acemoglu, D. (2009). Modern Economic Growth. Princeton, NJ: Princeton University Press.

[2] Aghion, P. \& Howitt, P. (1992). A Model of Growth through Creative Destruction. Econometrica, 60, 323-351.

[3] Aghion, P. \& Howitt, P. (1998). Endogenous Growth Theory. Cambridge, MA: MIT Press.

[4] Aghion, P. \& Howitt, P. (2009). The Economics of Growth. Cambridge, MA: MIT Press.

[5] Backus, D., Kehoe, P. \& Kehoe, T. (1992). In Search of Scale Effects in Trade and Growth. Journal of Economic Theory, 58(2), 377-409.

[6] Barro, R. \& Sala-i-Martin, X. (1995). Economic Growth. New York: McGraw-Hill.

[7] Bresnahan, T. \& Trajtenberg, M. (1995). General Purpose Technologies: "Engines of Growth"? Journal of Econometrics, 65(1), 83-108.

[8] Chantrel, E., Grimaud, A. \& Tournemaine, F. (2012). Pricing Knowledge and Funding Research of New Technology Sectors in a Growth Model. Journal of Public Economic Theory, 14(3), 493-520.

[9] Chari, V. \& Hopenhayn, H. (1991). Vintage Human Capital, Growth, and the Diffusion of New Technology. Journal of Political Economy, 99(6), 1142-1165. 
[10] Cozzi, G., Giordani, P.E. \& Zamparelli, L. (2007). The Refoundation of the Symmetric Equilibrium in Schumpeterian Growth Models. Journal of Economic Theory, 136(1) September, 788-797.

[11] Dinopoulos, E. \& Sener, F. (2007). New Directions in Schumpeterian Growth Theory. In H. Hanusch and A. Pyka (Eds.). Elgar Companion to Neo-Schumpeterian Economics, Edward Elgar.

[12] Dinopoulos, E. \& Thompson, P. (1998). Schumpeterian Growth Without Scale Effects. Journal of Economic Growth, 3, 313-335.

[13] Eicher, T. \& Turnovsky, S. (1999). Non-Scale Models of Economic Growth. Economic Journal, $109,394-415$.

[14] Gray, E. \& Grimaud, A. (2016). The Lindahl Equilibrium in Schumpeterian Growth Models. Journal of Evolutionary Economics, 26(1), 101-142.

[15] Griliches, Z. (1992). The Search for R\&D Spillovers. Scandinavian Journal of Economics, 94 (supplement), 29-47.

[16] Griliches, Z. (1995). R\&D and Productivity: Econometric Results and Measurement Issues. In P. Stoneman (Ed.), Handbook of the Economics of Innovation and Technical Change, Blackwell Handbooks in Economics.

[17] Grossman, G. \& Helpman, E. (1991). Quality Ladders in the Theory of Growth. Review of Economic Studies, 58, 43-61.

[18] Ha, J. \& Howitt, P. (2007). Accounting for Trends in Productivity and R\&D: A Schumpeterian Critique of Semi-Endogenous Growth Theory. Journal of Money, Credit and Banking, 39(4), 733-774.

[19] Hall, B. (2004). Innovation and Diffusion. In J. Fagerberg, D.C. Mowery and R.R. Nelson (Eds.), Handbook on Innovation. Oxford, Oxford University Press.

[20] Hall, B., Mairesse, J. \& Mohnen, P. (2010). Measuring the Returns to R\&D. In B. Hall and N. Rosenberg (Eds.), Handbook of the Economics of Innovation, Elsevier.

[21] Helpman, E. (Ed.) (1998). General Purpose Technologies and Economic Growth. Cambridge, MA: MIT Press.

[22] Howitt, P. (1999). Steady Endogenous Growth with Population and R\&D Inputs Growing. Journal of Political Economy, 107, 715-730.

[23] Jones, C. (1995a). Time Series Tests of Endogenous Growth Models. Quarterly Journal of Economics, 110, 495-525.

[24] Jones, C. (1995b). R\&D-based Models of Economic Growth. Journal of Political Economy, 103, 759-784.

[25] Jones, C. (1999). Growth: With or Without Scale Effects? American Economic Review, Papers and Proceedings, 89, 139-144.

[26] Jones, C. (2005). Growth and Ideas. In P. Aghion and S. Durlauf (Eds.), Handbook of Economic Growth (Volume 1B, pp. 1063-1111). Elsevier.

[27] Kortum, S. (1997). Research, Patenting, and Technological Change, Econometrica, 65, 13891419.

[28] Laincz, C. \& Peretto, P. (2006). Scale Effects in Endogenous Growth Theory: an Error of Aggregation not Specification. Journal of Economic Growth, 11, 263-288.

[29] Li, C.W. (2000). Endogenous vs. Semi-endogenous Growth in a Two-R\&D-sector Model. Economic Journal, 110, 109-122.

[30] Li, C.W. (2002). Growth and Scale Effects: the Role of Knowledge Spillovers. Economics Letters, 74, 177-185.

[31] Li, C.W. (2003). Endogenous Growth Without Scale Effects: A Comment. American Economic Review, 93(3), 1009-1017. 
[32] Madsen, J. (2008). Semi-Endogenous versus Schumpeterian Growth Models: Testing the Knowledge Production Function using International Data. Journal of Economic Growth, $13(1), 1-26$.

[33] Peretto, P. (1998). Technological Change and Population Growth. Journal of Economic Growth, 3(4), 283-311.

[34] Peretto, P. (1999). Cost Reduction, Entry, and the Interdependence of Market Structure and Economic Growth, Journal of Monetary Economics, 43, 173-195.

[35] Peretto, P. \& Smulders, S. (2002). Technological Distance, Growth and Scale Effects. Economic Journal, 112, 603-624.

[36] Romer, P. (1990). Endogenous Technological Change. Journal of Political Economy, 98(5), 71-102.

[37] Salop, S. (1979). Monopolistic Competition with Outside Goods. Bell Journal of Economics, $10,141-156$.

[38] Schulstad, P. (1993). Knowledge Diffusion in an Endogenous Growth Model. Economics Letters, $42,275-278$.

[39] Scotchmer, S. (1991). Standing on the Shoulders of Giants: Cumulative Research and the Patent Law. Journal of Economic Perspective, 5(1), 29-41.

[40] Segerstrom, P. (1998). Endogenous Growth Without Scale Effects. American Economic Review, 88, 1290-1310.

[41] Segerstrom, P. (2000). The Long-Run Growth Effects of R\&D Subsidies. Journal of Economic Growth, 5(3), 277-305.

[42] Smulders, S. \& van de Klundert, T. (1995). Imperfect Competition, Concentration and Growth with Firm-specific R\&D. European Economic Review, 39, 139-160.

[43] Weitzman, M.L. (1998). Recombinant Growth. Quarterly Journal of Economics, 113(2), 331360.

[44] Young, A. (1998). Growth Without Scale Effects. Journal of Political Economy, 106, 41-63. 